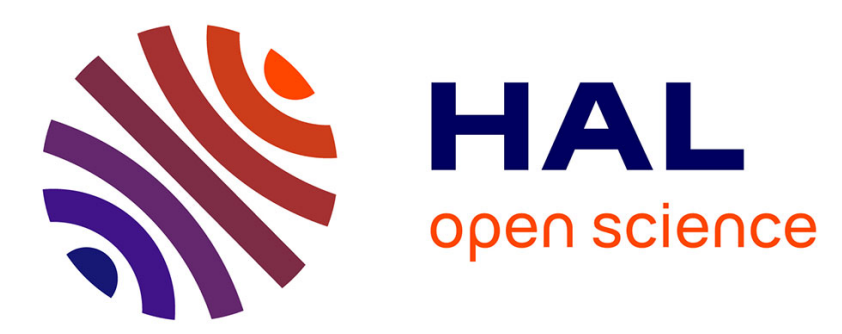

\title{
Homogenization of divergence-form operators with lower order terms in random media
}

\author{
Antoine Lejay
}

\section{To cite this version:}

Antoine Lejay. Homogenization of divergence-form operators with lower order terms in random media. Probability Theory and Related Fields, 2001, 120 (2), pp.255-276. 10.1007/s004400100135 . inria00001220

\section{HAL Id: inria-00001220 \\ https://hal.inria.fr/inria-00001220}

Submitted on 9 Apr 2006

HAL is a multi-disciplinary open access archive for the deposit and dissemination of scientific research documents, whether they are published or not. The documents may come from teaching and research institutions in France or abroad, or from public or private research centers.
L'archive ouverte pluridisciplinaire HAL, est destinée au dépôt et à la diffusion de documents scientifiques de niveau recherche, publiés ou non, émanant des établissements d'enseignement et de recherche français ou étrangers, des laboratoires publics ou privés. 


\title{
Homogenization of divergence-form operators with lower order terms in random media
}

\author{
Antoine Lejay ${ }^{1}$ — Projet SYSDYS (INRIA/LATP) \\ Abstract: The probabilistic machinery (Central Limit Theorem, \\ Feynman-Kac formula and Girsanov Theorem) is used to study \\ the homogenization property for PDE with second-order partial \\ differential operator in divergence-form whose coefficients are sta- \\ tionary, ergodic random fields. Furthermore, we use the theory \\ of Dirichlet forms, so that the only conditions required on the \\ coefficients are non degeneracy and boundedness.
}

Keywords: random media, random potential, homogenization, Dirichlet form, divergence-form operators

AMS Classification: 35B27 (31C25 35R60 60H30 60J60)

Published in Probability Theory and Related Fields. 120:2, pp. 255-276, 2001

Archives, links \& reviews:

○ DOI: $10.1007 / \mathrm{s} 004400100135$

○ MR Number: 1841330

${ }^{1}$ Current address: Projet OMEGA (INRIA Lorraine/IECN)

IECN, Campus scientifique

BP 239

54506 Vandœuvre-lès-Nancy CEDEX France

E-mail: Antoine.Lejay@iecn.u-nancy.fr 
A. Lejay / Homogenization of divergence-form operators in random media

\section{Introduction}

Some averaging — or homogenization — properties for some elliptic or parabolic partial differential equations (PDE) in a stationary, ergodic random media are studied with probabilistic techniques.

This consists in finding constant coefficients which approximate in some suitable sense highly oscillating coefficients that represent the random media. In other words, we study the limit of the solutions of some PDEs when a coefficient - which represents the scale of the heterogeneities - decreases to 0 .

Using the probabilistic representation of the solutions of parabolic and elliptic PDE, this leads to establishing a Central Limit Theorem for the stochastic process generated by a second-order partial differential operator.

More precisely, we are interested in PDEs with second-order partial differential operators of the form

$$
\begin{aligned}
A^{\varepsilon, \omega} & =L^{\varepsilon, \omega}+b_{i}(x / \varepsilon, \omega) \frac{\partial}{\partial x_{i}}+c(x / \varepsilon, \omega)+\frac{d(x / \varepsilon, \omega)}{\varepsilon}, \\
\text { where } L^{\varepsilon, \omega} & =\frac{e^{2 V(x / \varepsilon, \omega)}}{2} \frac{\partial}{\partial x_{i}}\left(a_{i, j}(x / \varepsilon, \omega) e^{-2 V(x / \varepsilon, \omega)} \frac{\partial}{\partial x_{j}}\right)
\end{aligned}
$$

under the assumption that the coefficients are bounded stationary random fields and that the matrix $a$ is symmetric. The operator $A^{\varepsilon, \omega}$ contains in fact a lower-differential term of the form $\partial_{x_{i}}\left(e_{i}(x / \varepsilon, \omega) \cdot\right)=e_{i}(x / \varepsilon, \omega) \partial_{x_{i}}+$ $\varepsilon^{-1}\left(\partial_{x_{i}} e_{i}\right)(x / \varepsilon, \omega)$, assuming that $e$ is differentiable.

The solution of the parabolic equation

$$
\frac{\partial u^{\varepsilon}(t, x)}{\partial t}=A^{\varepsilon, \omega} u^{\varepsilon}(t, x)
$$

with the initial condition $u^{\varepsilon}(0, \cdot)=f$ is given by the Feynman-Kac formula

$$
u^{\varepsilon}(t, x)=\widetilde{\mathbb{E}}_{x, \omega}^{\varepsilon}\left[\exp \left(\frac{1}{\varepsilon} \int_{0}^{t} d\left(\mathbf{X}_{s} / \varepsilon, \omega\right) \mathrm{d} s+\int_{0}^{t} c\left(\mathbf{X}_{s} / \varepsilon, \omega\right) \mathrm{d} s\right) f\left(\mathbf{X}_{t}\right)\right],
$$

where $\widetilde{\mathbb{E}}_{x, \omega}^{\varepsilon}$ is the distribution of the stochastic process generated by the operator $\widetilde{L}^{\varepsilon, \omega}=L^{\varepsilon, \omega}+b_{i}(x / \varepsilon, \omega) \partial_{x_{i}}$.

Studying of the convergence of the process $\widetilde{X}^{\varepsilon, \omega}$ associated to $\widetilde{L}^{\varepsilon, \omega}$ as $\varepsilon$ decreases to 0 is equivalent to studying the convergence of $\left(\varepsilon \cdot{ }^{\varepsilon} \widetilde{\mathbf{X}}_{t / \varepsilon^{2}}^{\omega}\right)_{t \geqslant 0}$, where ${ }^{\varepsilon} \mathbf{X}^{\omega}$ is the process whose infinitesimal generator is

$$
\frac{e^{-2 V(\cdot, \omega)}}{2} \frac{\partial}{\partial x_{i}}\left(a_{i, j}(\cdot, \omega) e^{2 V(\cdot, \omega)} \frac{\partial}{\partial x_{j}}\right)+\varepsilon b_{i}(x, \omega) \frac{\partial}{\partial x_{i}} .
$$


A. Lejay / Homogenization of divergence-form operators in random media

As it will be shown in Section 4, the Girsanov theorem allows to reduce this problem, where the first-order coefficient of the operator is of order $\varepsilon$, to the study of the Central Limit Theorem for the process $X^{1, \omega}$ whose generator is the self-adjoint divergence-form operator $L^{1, \omega}$. A rather similar use of the Girsanov transform in a different context to prove some homogenization results may be found in [23].

A Central Limit Theorem for the process $\int_{0}^{\cdot} d\left(\mathbf{X}_{s}^{\omega}\right) \mathrm{d} s$ has to be proved to deal with our initial problem (i.e., with a highly-oscillating zero-order term).

The homogenization property for the divergence-form operator $\partial_{x_{i}}\left(a_{i, j}(\cdot / \varepsilon, \omega) \partial_{x_{j}}\right)$ with random coefficients has been studied with analytical tools first by S. Kozlov [17, 18] and G. Papanicolaou and S.R.S. Varadhan [34] (see also [35]).

The probabilistic method consists in finding functions which are solutions of auxiliary problems, so that our process is transformed as the sum of a local martingale and a process that converges to 0. Then, the Central Limit Theorem for the local martingale is applied with the help of the Ergodic Theorem. See e.g., [10, 3, 16] for various applications of this procedure.

The difficulty for homogenization in random media lies in the resolution of the auxiliary problem, that has to be done on a suitable space.

For a general random media - in contradistinction to what happens in the case of periodic media where the Poincaré inequality holds (see e.g., [24] for results under weaker hypotheses in periodic media) - , the resolution of the auxiliary problem cannot be considered in a direct way, because the needed function is not a stationary random fields. Three strategies may be used: 1) The solution is approximated by a sequence of stationary random fields, and one studies the convergence of their gradients. This method is especially well-suited to the case of an initial environment whose law is the invariant distribution. (see e.g., [6, 29, 13, 31]). 2) The solution is directly constructed using the spectral theory for the shift operators of the random media (see e.g., [34, 9]). 3) The gradient of the solution is given directly in an appropriate space with the help of the Lax-Milgram Theorem (see [15] for an analytical use of this method).

The two last approaches may be used to prove that the family of processes converges for almost every realization. We have chosen here to use the third method. Our approach is close to that used by S. Kozlov in [19] for random walks.

Furthermore, this approach does not really relies on the idea of mean forward velocity as in [30, 6, 13, 31]. Our proof of the Central Limit Theorem for the process associated to $L^{1, \omega}$ is then rather different to that of [31, which itself adapts to Dirichlet forms the ideas developed in [16, 6, 29, 13.

Solving the auxiliary problem or finding the invariant measure shows the 
A. Lejay / Homogenization of divergence-form operators in random media

difficulty to study the limit behaviour of the processes associated to

$$
\frac{1}{2} \frac{\partial}{\partial x_{i}}\left(a_{i, j}(x / \varepsilon, \omega) \frac{\partial}{\partial x_{j}}\right)+\frac{1}{\varepsilon} b_{i}(x / \varepsilon, \omega) \frac{\partial}{\partial x_{i}}
$$

for a general $b$, which so far remains an open problem. In fact, there exists some counterexamples to the homogenization property for some stationary, divergence-free random fields [1, 14]. We have also assumed that $d$ is the derivative of a bounded function.

Although some results may be given for general non-symmetric Dirichlet forms [32] provided the mean forward velocity exists, two classes of problems are generally considered: The first concerns the case where $b$ is the derivative of the skew-symmetric matrix which is a stationary random field [28, 9, 22, 34. The second concerns the case where $b$ is a gradient of a stationary random field. In this case, the second-order differential operator is reduced to a self-adjoint operator.

The term $V$ is a potential. If $a$ and $V$ are regular enough, then the operator $L^{\varepsilon, \omega}$ can be written

$$
L^{\varepsilon, \omega}=\frac{1}{2} a_{i, j}(\cdot / \varepsilon, \omega) \frac{\partial^{2}}{\partial x_{i} \partial x_{j}}+\frac{1}{2 \varepsilon}\left(\frac{\partial a_{i, j}}{\partial x_{j}}-2 a_{i, j} \frac{\partial V}{\partial x_{j}}\right)(\cdot / \varepsilon, \omega) \frac{\partial}{\partial x_{i}}
$$

and a stochastic process may be associated to $L^{\varepsilon, \omega}$ via the theory of stochastic differential equations.

However, any regularity assumption on $a$ and $V$ may be dropped if one use the theory of Dirichlet forms as developed e.g., in 11] instead of Itô stochastic calculus. Hence, our results generalize those of [6, Section 6] and [29, Chapter 2]. In fact, our proofs use some considerations on the semi-group associated to a divergence-form operator, but hardly require the theory of Dirichlet forms.

Afterwards, we prove that the solution to the parabolic PDE (3) converges to the solution to the parabolic PDE

$$
\frac{\partial u(t, x)}{\partial t}=\bar{A} u(t, x)
$$

where the coefficients of the PDE operator $\bar{A}$ are constant and are averages of the coefficients of $A^{1, \omega}$ with respect to the law of the media. We use the method introduced by É. Pardoux in [33] to deal with the highly-oscillating zero-order term, which also uses the Girsanov theorem.

In Section 5.2, we consider the case of the elliptic equations of the form $\left(\alpha-A^{\varepsilon, \omega}\right) u^{\varepsilon}=f$. We prove that $\alpha-A^{\varepsilon, \omega}$ is invertible for $\alpha$ greater to some 
A. Lejay / Homogenization of divergence-form operators in random media

value $\alpha_{0}$ that does not depend on $\varepsilon$ nor $\omega$. Provided that $\alpha \geqslant \alpha_{0}$, the solution $u^{\varepsilon}$ converges to the solution of $(\alpha-\bar{A}) u=f$, with $\bar{A}$ as above.

The method to deal with first-order and highly oscillatory zero-order terms may be easily used to solve some other homogenization problems (e.g., the operators of the forms $\Delta+\frac{1}{\varepsilon} b(\cdot / \varepsilon, \omega)$, where $b$ is divergence-free).

In Section 2, we recall some generalities about random media and the construction of the environment viewed from the particle. Section 3 contains our proof of the homogenization property both for the operator $L^{\varepsilon, \omega}$ and the additive functional $\int_{0}^{\cdot} d\left(\mathbf{X}_{s}\right) \mathrm{d} s$. These results are extended to operators with a first-order differential term in Section 4. The connection of the probabilistic proof of the convergence and the parabolic or elliptic PDEs is studied in Section 5, We conclude with some immediate generalizations of our results.

\section{The environment viewed from the particle}

\section{$2.1 \quad$ Random media}

A random media is a probability space $(\Omega, \mathcal{G}, \mu)$ on which is defined a group $\left(\tau_{x}\right)_{x \in \mathbb{R}^{n}}$ of transformations acting on $\Omega$ such that

$(\mathrm{MP}) \mu\left(\tau_{x} A\right)=\mu(A)$ for any $A$ in $\mathcal{G}$ and any $x \in \mathbb{R}^{n}$;

(ERG) if $\tau_{x} A=A$ for any $x \in \mathbb{R}^{n}$, then $\mu(A)=0$ or 1 ;

(MEAS) for any measurable function $\mathbf{f}$ on $(\Omega, \mathcal{G}, \mu)$, the function $(x, \omega) \mapsto$ $\mathbf{f}\left(\tau_{x} \omega\right)$ is measurable on $\left(\mathbb{R}^{n} \otimes \Omega, \operatorname{Bor}\left(\mathbb{R}^{n}\right) \otimes \mathcal{G}\right)$;

(SC) for any $\mathbf{f}$ in the space of square integrable functions $\mathrm{L}^{2}(\mu)$ on $(\Omega, \mathcal{G}, \mu)$

$$
\mu\left\{\left|\mathbf{f}\left(\tau_{h} \omega\right)-\mathbf{f}(\omega)\right| \geqslant \delta\right\} \underset{|h| \rightarrow 0}{\longrightarrow} 0
$$

for any $\delta>0$. The group $\left(\tau_{x}\right)_{x \in \mathbb{R}^{n}}$ is said to be stochastically continuous.

To any function $\mathbf{f}$ on $(\Omega, \mathcal{G}, \mu)$ is associated a stationary random field $f$, which is a measurable function on $\left(\mathbb{R}^{n} \times \mathcal{G}\right.$, Bor $\left.\left(\mathbb{R}^{n}\right) \otimes \mathcal{G}\right)$ defined by $f(x, \omega)=$ $\mathbf{f}\left(\tau_{x} \omega\right)$. We use bold letters to denote functions on $\Omega$, while their italic counterparts denote stationary random fields.

If $\mathbf{f}$ belongs to $\mathrm{L}^{p}(\mu)(p \geqslant 1)$, which is the space of random variables on $\Omega$ such that $\int_{\Omega}|\mathbf{f}|^{p} \mathrm{~d} \mu$ is finite, then almost every realization of $\mathbf{f}$ belongs the space $\mathrm{L}_{\text {loc }}^{p}\left(\mathbb{R}^{n}\right)$ of functions whose $p^{\text {th }}$-power is locally integrable. 
The group $\left(T_{x}\right)_{x \in \mathbb{R}^{n}}$ of unitary operators on $\mathrm{L}^{2}(\mu)$ defined by by $T_{x} \mathbf{f}(\omega)=$ $\mathbf{f}\left(\tau_{x} \omega\right)$ is strongly continuous, because of the stochastic continuity condition (SC) of $\left(\tau_{x}\right)_{x \in \mathbb{R}^{n}}$. Its $n$ infinitesimal generators $D_{1}, \ldots, D_{n}$ are defined by

$$
D_{i} \mathbf{f}=\lim _{h \rightarrow 0} \frac{T_{h e_{i}} \mathbf{f}-\mathbf{f}}{h} \text { when this limit exists in } \mathrm{L}^{2}(\mu),
$$

where $\left(e_{1}, \ldots, e_{n}\right)$ is the canonical basis of $\mathbb{R}^{n}$. These operators are closed and densely defined.

For any $\mathbf{f}$ in $L^{2}(\mu)$ and any smooth function $\varphi$ with compact support, the operation

$$
\mathbf{f} \star \varphi(\omega)=\int_{\mathbb{R}^{n}} \mathbf{f}\left(\tau_{x} \omega\right) \varphi(x) \mathrm{d} x
$$

defines a function in $\mathrm{L}^{2}(\mu)$. This operation $\star$ has to be seen as a convolution.

Let $\mathcal{C}$ be the set

$$
\mathcal{C}=\left\{\mathbf{f} \star \varphi \mid \mathbf{f} \in \mathrm{L}^{2}(\mu), \varphi \in \mathcal{C}_{\mathrm{c}}^{\infty}\left(\mathbb{R}^{n} ; \mathbb{R}\right)\right\} .
$$

This space is dense in $\mathrm{L}^{2}(\mu)$ (see $e . g .,[22]$ ). Let us denote by $\langle\cdot, \cdot\rangle_{\mu}$ the usual scalar product on $\mathrm{L}^{2}(\mu)$. Let $\mathcal{H}^{1}(\mu)$ be the closure of $\mathcal{C}$ with respect to the norm associated to the scalar product

$$
\langle\mathbf{f}, \mathbf{g}\rangle_{\mathcal{H}^{1}(\mu)}=\langle\mathbf{f}, \mathbf{g}\rangle_{\mu}+\left\langle D_{i} \mathbf{f}, D_{i} \mathbf{g}\right\rangle_{\mu}
$$

This space $\mathcal{C}$ plays the role of smooth functions for the set of functions on $(\Omega, \mathcal{G}, \mu)$. Using the condition of invariance of $\left(\tau_{x}\right)_{x \in \mathbb{R}^{n}}$ with respect to $\mu$,

$$
\langle\mathbf{g}, \mathbf{f} \star \varphi\rangle_{\mu}=\int_{\Omega} \mathbf{f}(\omega) \int_{\mathbb{R}^{n}} g(x, \omega) \varphi(-x) \mathrm{d} x \mathrm{~d} \mu .
$$

This relation is particularly useful, because it allows to switch between formulation given for random variables, and formulations given for stationary random fields for almost every realization.

\subsection{Divergence-form operator}

We assume in first time that the first-order differential term $b$ is equal to 0 . The following hypothesis are assumed on the coefficients of $L^{\varepsilon, \omega}$ defined by (2).

Hypothesis 1. The matrix-valued function $\mathbf{a}=\left(\mathbf{a}_{i, j}\right)_{i, j=1}^{n}$ is measurable on $(\Omega, \mathcal{G}, \mu)$ and $\mathbf{a}(\cdot)$ is a symmetric matrix. Furthermore, we assume that there exist two positive constants $\lambda$ and $\Lambda$ such that

$$
\forall \xi \in \mathbb{R}^{n}, \lambda|\xi|^{2} \leqslant\langle\mathbf{a}(\omega) \xi, \xi\rangle \leqslant \Lambda|\xi|^{2}, \mu \text {-a.s.. }
$$


A. Lejay / Homogenization of divergence-form operators in random media

The measurable function $\mathbf{V}$ is bounded by $\Lambda$ on $(\Omega, \mathcal{G}, \mu)$ and we may assume without loss of generality that

$$
\int_{\Omega} e^{-2 \mathbf{V}(\omega)} \mathrm{d} \mu(\omega)=1
$$

Using our notations, the stationary random fields corresponding to a and $\mathbf{V}$ are denoted by $a=\left(a_{i, j}\right)_{i, j=1}^{n}$ and $V$.

Let us consider the family of Dirichlet forms on $\mathrm{L}^{2}\left(\mathbb{R}^{n}, e^{-2 V(x, \omega)} \mathrm{d} x\right)$ defined by

$$
\mathcal{E}^{\varepsilon, \omega}(u, v)=\frac{1}{2} \int_{\mathbb{R}^{n}} a_{i, j}(x / \varepsilon, \omega) \frac{\partial u(x)}{\partial x_{i}} \frac{\partial v(x)}{\partial x_{j}} e^{-2 V(x / \varepsilon, \omega)} \mathrm{d} x
$$

with domain $\mathrm{H}^{1}\left(\mathbb{R}^{n}\right)$. For each $\omega \in \Omega$ and each $\varepsilon>0$, there exists a unique self-adjoint operator $\left(L^{\varepsilon, \omega}, \operatorname{Dom}\left(L^{\varepsilon, \omega}\right)\right)$ such that

$$
\mathcal{E}^{\varepsilon, \omega}(u, v)=-\left\langle L^{\varepsilon, \omega} u, v\right\rangle_{\mathrm{L}^{2}\left(\mathbb{R}^{n}\right)}, \forall(u, v) \in \operatorname{Dom}\left(L^{\varepsilon, \omega}\right) \times \mathrm{H}^{1}\left(\mathbb{R}^{n}\right) .
$$

It is well known that there exists a weak solution, - called the fundamental solution, - to the equation

$$
\left\{\begin{array}{l}
\frac{\partial p^{\varepsilon}(\omega, t, x, y)}{\partial t}=L^{\varepsilon, \omega} p^{\varepsilon}(\omega, t, x, y) \\
p^{\varepsilon}(\omega, 0, x, y)=\delta_{x-y}, \forall y \in \mathbb{R}^{n}
\end{array}\right.
$$

where $\delta_{x-y}$ is the Dirac mass at the point $x-y$. This solution $p^{\varepsilon}(\omega, t, x, y)$ corresponds to the density of the semi-group $\left(P_{t}^{\varepsilon, \omega}\right)_{t>0}$ (see e.g., [2, 21]).

One remarkable results about the density is that it satisfies the Aronson Estimate:

$$
\frac{1}{M t^{n / 2}} \exp \left(\frac{-M|x-y|^{2}}{t}\right) \leqslant p^{\varepsilon}(\omega, t, x, y) \leqslant \frac{M}{t^{n / 2}} \exp \left(\frac{-|x-y|^{2}}{M t}\right)
$$

for any $(t, x, y)$ in $\mathbb{R}_{+}^{*} \times \mathbb{R}^{n} \times \mathbb{R}^{n}$, where the constant $M$ depends only on $\lambda$, $\Lambda$ and $n$. It is also known that there exist two constants $C$ and $\alpha$ depending only on $\lambda, \Lambda$ and $n$ such that for every $\delta>0$,

$$
\left|p^{\varepsilon}\left(\omega, t^{\prime}, x^{\prime}, y^{\prime}\right)-p^{\varepsilon}(\omega, t, x, y)\right| \leqslant \frac{C}{\delta^{n}}\left(\frac{\sqrt{\left|t^{\prime}-t\right|} \vee\left|x^{\prime}-x\right| \vee\left|y^{\prime}-y\right|}{\delta}\right)^{\alpha}
$$

for all $\left(t^{\prime}, x^{\prime}, y^{\prime}\right),(t, x, y) \in\left[\delta^{2},+\infty\right) \times \mathbb{R}^{n} \times \mathbb{R}^{n}$ with $\left|y^{\prime}-y\right| \vee\left|x^{\prime}-x\right| \leqslant \delta$. A proof of these estimates may be found in [38]. 
Setting $P_{t} f(x)=\int_{\mathbb{R}^{n}} p(t, x, y) f(y) \mathrm{d} y$, it may be proved with (5) and (6) that $P_{t}$ maps $\mathrm{L}^{2}\left(\mathbb{R}^{n}\right)$ into the space of continuous functions that vanish at infinity.

Hence, the semi-group $\left(P_{t}^{\varepsilon, \omega}\right)_{t>0}$ is a Feller semi-group, and there exists a continuous conservative Hunt process $\left(X, \mathbb{P}_{x, \omega}^{\varepsilon}\right)_{x \in \mathbb{R}^{n}}$ whose generator is $L^{\varepsilon, \omega}$ with domain $\left.\operatorname{Dom}\left(L^{\varepsilon, \omega}\right)\right)$. Such a result is stated e.g., in Theorem 1.9.4 in [4] or in [7, Theorem 2.7, p. 169].

The key tool for studying such a process is the Theory of Dirichlet forms: see [11, 27] for example.

From now, we denote by $X$ is the canonical process on the space of continuous functions, i.e., $\mathrm{X}_{t}(\omega)=\omega_{t}$ for any $\omega$ in $\mathcal{C}\left([0,+\infty) ; \mathbb{R}^{n}\right)$.

\subsection{The environment viewed from the particle}

The aim of this paragraph is to construct the process

$$
\left\{\begin{array}{l}
Y_{t}^{\omega}=\tau_{\mathbf{X}_{t}^{1, \omega}} \omega, \text { for } t>0 \\
Y_{0}^{\omega}=\omega
\end{array}\right.
$$

where $\mathrm{X}^{1, \omega}$ is the process with generator $\left(L^{1, \omega}, \operatorname{Dom}\left(L^{1, \omega}\right)\right)$ and such that $X^{1, \omega}=0$. We denote by $\mathbb{P}_{\omega}$ the distribution of $Y^{\omega}$. We also prove that $Y^{\omega}$ is ergodic for the distribution $\int_{\Omega} e^{-2 \mathbf{V}(\omega)} \mathbb{P}_{\omega}[\cdot] \mathrm{d} \mu$.

Let $\left(G_{\alpha}^{\omega}\right)_{\alpha>0}$ be the resolvent of the Dirichlet form $\mathcal{E}^{1, \omega}$. For any $\alpha>0$, the operator $G_{\alpha}^{\omega}$ may be written $G_{\alpha}^{\omega} f(x)=\int_{\mathbb{R}^{n}} g_{\alpha}(\omega, x, y) f(y) \mathrm{d} y$ for any $f$ in $\mathrm{L}^{2}\left(\mathbb{R}^{n}\right)$, with $g_{\alpha}(\omega, x, y)=\int_{0}^{+\infty} e^{-\alpha t} p^{\varepsilon}(\omega, t, x, y) \mathrm{d} t$.

If $f$ is a stationary random field in $\mathrm{L}_{\text {loc }}^{p}\left(\mathbb{R}^{n}\right)$ with $p=1,2, G_{\alpha}^{\omega} f(x, \omega)$ is also a stationary random field in $\mathrm{L}_{\text {loc }}^{p}\left(\mathbb{R}^{n}\right)$. As a result, we set

$$
\mathbf{G}_{\alpha} \mathbf{f}(\omega)=\int_{\mathbb{R}^{n}} g_{\alpha}(\omega, 0, y) \mathbf{f}\left(\tau_{y} \omega\right) \mathrm{d} y .
$$

Let $\mathrm{L}^{2}(\pi)$ be the Hilbert space equipped with the scalar product

$$
\langle\mathbf{u}, \mathbf{v}\rangle_{\pi}=\int_{\Omega} \mathbf{u} \mathbf{v} e^{-2 \mathbf{v}} \mathrm{d} \mu .
$$

The closed bilinear form and densely defined on $\mathrm{L}^{2}(\pi)$ corresponding to the Dirichlet form $\mathcal{E}^{1, \omega}$ on $(\Omega, \mathcal{G}, \mu)$ is

$$
\mathcal{E}^{\pi}(\mathbf{u}, \mathbf{v})=\int_{\Omega} \mathbf{a}_{i, j} D_{i} \mathbf{u} D_{j} \mathbf{v} e^{-2 \mathbf{v}} \mathrm{d} \mu \forall \mathbf{u}, \mathbf{v} \in \mathcal{H}^{1}(\mu) .
$$

Lemma 1. The family $\left(\mathbf{G}_{\alpha}\right)_{\alpha>0}$ is the resolvent of the process $\left(\mathbf{Y}_{t}^{\omega}, t \geqslant\right.$ $\left.0 ; \mathbb{P}_{\omega}\right)_{\omega \in \Omega}$. 
A. Lejay / Homogenization of divergence-form operators in random media

Proof. For any $\mathbf{u}$ in $\mathrm{L}^{2}(\mu)$,

$$
\begin{aligned}
& \int_{\Omega} \mathcal{E}^{1, \omega}\left(G_{\alpha}^{\omega} f(\cdot, \omega), \varphi\right) \mathbf{u}(\omega) \mathrm{d} \mu(\omega) \\
& \quad+\alpha \int_{\Omega \times \mathbb{R}^{n}} G_{\alpha}^{\omega} f(x, \omega) \varphi(x) \mathbf{u}(\omega) e^{-2 V(x, \omega)} \mathrm{d} x \mathrm{~d} \mu(\omega) \\
& \quad=\int_{\Omega \times \mathbb{R}^{n}} f(x, \omega) \varphi(x) \mathbf{u}(\omega) e^{-2 V(x, \omega)} \mathrm{d} x \mathrm{~d} \mu(\omega) .
\end{aligned}
$$

If $\check{\varphi}(x)=\varphi(-x)$ and $\mathbf{G}_{\alpha} \mathbf{f}\left(\tau_{x} \omega\right)=G_{\alpha}^{\omega} f(x, \omega)$, the former equation is equivalent to

$$
\mathcal{E}^{\pi}\left(\mathbf{G}_{\alpha} \mathbf{f}, \mathbf{u} \star \check{\varphi}\right)+\alpha\left\langle\mathbf{G}_{\alpha} \mathbf{f}, \mathbf{u} \star \check{\varphi}\right\rangle_{\pi}=\langle\mathbf{f}, \mathbf{u} \star \check{\varphi}\rangle_{\pi} .
$$

The last equation is true for any smooth function $\varphi$ with compact support and any $\mathbf{u}$ in $\mathrm{L}^{2}(\mu)$. By density of $\mathcal{C}$ in $\mathrm{L}^{2}(\mu),\left(\mathbf{G}_{\alpha}\right)_{\alpha>0}$ is the resolvent of $\left(\mathcal{E}^{\pi}, \mathcal{H}^{1}(\mu)\right)$.

We also deduce that

$$
G_{\alpha}^{\omega} f(0, \omega)=\int_{0}^{+\infty} e^{-\alpha t} \mathbb{E}_{0, \omega}^{1}\left[f\left(\mathrm{X}_{t}, \omega\right)\right] \mathrm{d} t=\int_{0}^{+\infty} e^{-\alpha t} \mathbb{E}_{\omega}\left[\mathbf{f}\left(\mathrm{Y}_{t}^{\omega}\right)\right] \mathrm{d} t=\mathbf{G}_{\alpha} \mathbf{f}(\omega) .
$$

Hence, $\left(\mathbf{G}_{\alpha}\right)$ is also the resolvent of the process $\mathrm{Y}^{\omega}$.

Lemma 2. The process $\mathrm{Y}^{\omega}$ is ergodic with respect to the measure $e^{-2 \mathbf{V}} \mu$.

Proof. We know that $\mathrm{Y}^{\omega}$ is ergodic when the only functions in $\mathrm{L}^{2}(\mu)$ such that $\alpha \mathbf{G}_{\alpha} \mathbf{f}=\mathbf{f}$ are the constants [20, Theorem 1.3.7, p. 22]. But if $\alpha \mathbf{G}_{\alpha} \mathbf{f}=\mathbf{f}$, then clearly $\mathcal{E}^{\pi}(\mathbf{f}, \mathbf{f})=0$. This implies that $D_{i} \mathbf{f}=0, \mu$-a.s. for $i=1, \ldots, n$. The operator $\star$ allows to proves rigorously that $\mathbf{f}$ is constant. For any smooth function $\varphi$ with compact support and for $i=1, \ldots, n$,

$$
0=-D_{i} \mathbf{f} \star \varphi=\mathbf{f} \star \frac{\partial \varphi}{\partial x_{i}}=\int_{\mathbb{R}^{n}} f(x, \omega) \frac{\partial \varphi(x)}{\partial x_{i}} \mathrm{~d} x, \mu \text {-a.s. },
$$

which proves that for almost every realization, the function $f(\cdot, \omega)$ is just a function of $\omega$. But, with the hypothesis of ergodicity (ERG), f is constant.

Clearly,

$$
\alpha\left\langle\mathbf{G}_{\alpha} \mathbf{u}, 1\right\rangle_{\pi}+\mathcal{E}^{\pi}\left(\mathbf{G}_{\alpha} \mathbf{u}, 1\right)=\alpha\left\langle\mathbf{G}_{\alpha} \mathbf{u}, e^{-2 \mathbf{V}}\right\rangle_{\mu}=\left\langle\mathbf{u}, e^{-2 \mathbf{V}}\right\rangle_{\mu}, \forall \mathbf{u} \in \mathrm{L}^{2}(\mu) .
$$

Hence, $e^{-2 \mathbf{V}} \mathrm{d} \mu$ is the invariant, ergodic measure for $\mathrm{Y}^{\omega}$.

Remark 1. We choose here to use a bilinear form and the resolvent, but we also may have construct a "differential" operator and the corresponding semi-group as in [29]. 
A. Lejay / Homogenization of divergence-form operators in random media

\section{Homogenization of the divergence-form op- erator}

We still assume that the first-differential order term $\mathbf{b}$ is equal to 0 . Let $\mathbf{d}$ and $\mathbf{c}$ be some bounded measurable functions on $(\Omega, \mathcal{G}, \mu)$. These terms give the zero-order terms of the partial differential operator $A^{\varepsilon, \omega}$ defined by (11) and an additional condition is required on $\mathbf{d}$.

Hypothesis 2. There exists some some bounded functions $\widehat{\mathbf{d}}_{1}, \ldots, \widehat{\mathbf{d}}_{n}$ in $\mathcal{H}^{1}(\mu)$ such that

$$
e^{-2 \mathbf{V}} \mathbf{d}=D_{1} \widehat{\mathbf{d}}_{1}+\cdots+D_{n} \widehat{\mathbf{d}}_{n}
$$

This hypothesis implies that $\int_{\Omega} \mathbf{d}(\omega) e^{-2 \mathbf{V}(\omega)} \mathrm{d} \mu(\omega)=0$.

We set

$$
V_{t}^{\varepsilon, \omega}\left(\mathbf{X}^{\varepsilon, \omega}\right)=\int_{0}^{t}\left(\frac{1}{\varepsilon} d\left(\mathbf{X}_{s}^{\varepsilon, \omega} / \varepsilon, \omega\right)+c\left(\mathbf{X}_{s}^{\varepsilon, \omega} / \varepsilon, \omega\right)\right) \mathrm{d} s .
$$

We prove now the joint convergence of $\mathbf{X}^{\varepsilon, \omega}$ and $V^{\varepsilon, \omega}\left(\mathbf{X}^{\varepsilon, \omega}\right)$.

Proposition 1. Under Hypotheses 1 and [2, there exist some symmetric matrix $\bar{a}=\left(\bar{a}_{i, j}\right)_{i, j=1^{n}}$ together with some reals $\bar{c}=\int_{\Omega} \mathbf{c} e^{-2 \mathbf{V}} \mathrm{d} \mu$ and $\bar{D}$ depending respectively on the coefficients of $L^{\varepsilon, \omega}$ and $A^{\varepsilon, \omega}$ (see formula (16)) such that for almost every environment $\omega \in \Omega$,

$$
\mathcal{L}\left(\mathrm{X}^{1}, \ldots, \mathrm{X}^{n}, V^{\varepsilon, \omega}(\mathrm{X}) \mid \mathbb{P}_{0, \omega}^{\varepsilon}\right) \underset{\varepsilon \rightarrow 0}{\longrightarrow} \mathcal{L}\left(\overline{\mathrm{M}}_{t}^{1}, \ldots, \overline{\mathrm{M}}_{t}^{n}, \overline{\mathrm{M}}_{t}^{n+1}+\bar{c} t, t \geqslant 0 \mid \mathbb{P}\right),
$$

where $(\overline{\mathrm{M}}, \mathbb{P})$ is a $(n+1)$-dimensional non-standard Brownian motion $\overline{\mathrm{M}}$ characterized by

$$
\begin{cases}\left\langle\overline{\mathrm{M}}^{i}, \overline{\mathrm{M}}^{j}\right\rangle_{t}=\bar{a}_{i, j} t, \quad \text { if } 1 \leqslant i, j \leqslant n, \\ \left\langle\overline{\mathrm{M}}^{n+1}, \overline{\mathrm{M}}^{i}\right\rangle_{t}=0, \quad \text { if } 1 \leqslant i \leqslant n, \\ \left\langle\overline{\mathrm{M}}^{n+1}\right\rangle=\bar{D} t .\end{cases}
$$

Let us defined the auxiliary problems. For that, we need to work on some appropriate space.

Let $\mathcal{V}_{\text {pot }}^{2}$ be the Hilbert space

$$
\begin{aligned}
& \mathcal{V}_{\text {pot }}^{2}=\left\{\begin{array}{l|l}
\left(\mathbf{f}_{1}, \ldots, \mathbf{f}_{n}\right) \in \mathrm{L}^{2}(\mu)^{n} & \begin{array}{l}
\int_{\Omega} \mathbf{f}_{i} \mathrm{~d} \mu=0, \mathbf{f}_{i} \star \frac{\partial \varphi}{\partial x_{j}}=\mathbf{f}_{j} \star \frac{\partial \varphi}{\partial x_{i}}, \\
\forall \varphi \in \mathcal{C}_{\mathrm{c}}^{\infty}\left(\mathbb{R}^{n} ; \mathbb{R}\right), i, j=1, \ldots, n
\end{array}
\end{array}\right\}
\end{aligned}
$$

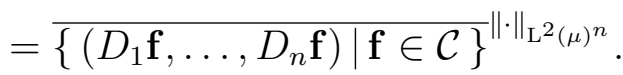


A. Lejay / Homogenization of divergence-form operators in random media

This space is equipped with the scalar product $\langle\cdot, \cdot\rangle_{\mathrm{L}^{2}(\mu)^{n}}$. The second identification for the space $\mathcal{V}_{\text {pot }}^{2}$ follows from the fact that, if

$$
\mathrm{L}_{\text {sol }}^{2}=\left\{\left(\mathbf{f}_{1}, \ldots, \mathbf{f}_{n}\right) \in \mathrm{L}^{2}(\mu)^{n} \mid \sum_{j=1}^{n} \mathbf{f}_{j} \star \frac{\partial \varphi}{\partial x_{j}}=0, \forall \varphi \in \mathcal{C}_{\mathrm{c}}^{\infty}\left(\mathbb{R}^{n} ; \mathbb{R}\right)\right\} .
$$

then $\mathrm{L}^{2}(\mu)^{n}=\mathcal{V}_{\text {pot }}^{2} \oplus \mathrm{L}_{\text {sol }}^{2}$. This is the Weyl Decomposition [15, p. 230].

Let $\mathcal{G}^{\pi}$ be the bilinear form defined by

$$
\mathcal{G}^{\pi}(\mathbf{f}, \mathbf{g})=\frac{1}{2} \int_{\Omega} e^{-2 \mathbf{V}(\omega)} \mathbf{a}_{i, j}(\omega) \mathbf{f}_{i}(\omega) \mathbf{g}_{j}(\omega) \mathrm{d} \mu(\omega)
$$

for $\mathbf{f}=\left(\mathbf{f}_{1}, \ldots, \mathbf{f}_{n}\right)$ and $\mathbf{g}=\left(\mathbf{g}_{1}, \ldots, \mathbf{g}_{n}\right)$ in $\mathcal{V}_{\text {pot }}^{2}$. The choice of $\mathcal{G}^{\pi}$ comes from that for any $\mathbf{u}, \mathbf{v}$ in $\mathcal{C}$,

$$
\mathcal{G}^{\pi}(\nabla \mathbf{u}, \nabla \mathbf{v})=\mathcal{E}^{\pi}(\mathbf{u}, \mathbf{v}) \text { where } \nabla=\left(D_{1}, \ldots, D_{n}\right) .
$$

Now, $\mathcal{G}^{\pi}$ is coercive and continuous on $\mathcal{V}_{\text {pot }}^{2}$. From the Lax-Milgram Theorem, there exists for $i=1, \ldots, n$ a unique solution $\left(\mathbf{f}_{1}^{i}, \ldots, \mathbf{f}_{n}^{i}\right)$ in $\mathcal{V}_{\text {pot }}^{2}$ to

$$
\mathcal{G}^{\pi}\left(\mathbf{f}^{i}, \mathbf{u}\right)=\frac{-1}{2} \int_{\Omega} \mathbf{a}_{i, j} e^{-2 \mathbf{V}} \mathbf{u}_{j} \mathrm{~d} \mu, \forall\left(\mathbf{u}_{1}, \ldots, \mathbf{u}_{n}\right) \in \mathcal{V}_{\text {pot }}^{2} .
$$

Let $\left(\mathbf{g}_{1}, \ldots, \mathbf{g}_{n}\right)$ be the solution in $\mathcal{V}_{\text {pot }}^{2}$ of

$$
\mathcal{G}^{\pi}(\mathbf{g}, \mathbf{u})=\int_{\Omega} \widehat{\mathbf{d}}_{i} \mathbf{u}_{i} \mathrm{~d} \mu, \forall\left(\mathbf{u}_{1}, \ldots, \mathbf{u}_{n}\right) \in \mathcal{V}_{\text {pot }}^{2} .
$$

Remark 2. The elements $\left(\widehat{\mathbf{d}}_{1}, \ldots, \widehat{\mathbf{d}}_{n}\right)$ are defined up to an element $\left(\mathbf{u}_{1}, \ldots, \mathbf{u}_{n}\right)$ in $\mathrm{L}_{\text {sol }}^{2}$. But the Weyl decomposition implies that $\mathbf{g}$ is unique.

Definition 1 (Auxiliary problems). The problems (10) and (11) are called auxiliary problems.

We set $v_{i}(x)=\int_{0}^{1} x_{j} f_{j}^{i}(t x, \omega) \mathrm{d} t$, in order that $\partial_{x_{j}} v_{i}(x, \omega)=\mathbf{f}_{j}^{i}\left(\tau_{x} \omega\right)$. The function $u_{i}^{\varepsilon}(t, x)=x_{i}+\varepsilon v_{i}(x / \varepsilon, \omega)$ is harmonic for $L^{\varepsilon, \omega}$, i.e., $L^{\varepsilon, \omega} u^{\varepsilon}(\cdot, \omega)=0$ in the weak sense.

We set $h(x, \omega)=\int_{0}^{1} x_{i} g_{i}(t x, \omega) \mathrm{d} t$ and $h^{\varepsilon}(x, \omega)=\varepsilon h(x / \varepsilon, \omega)$, so that

$$
\begin{aligned}
\mathcal{E}^{\varepsilon, \omega}\left(h^{\varepsilon}(\cdot, \omega), \varphi\right) & =\int_{\mathbb{R}^{n}} \frac{\partial \widehat{d}_{i}}{\partial x_{i}}(x / \varepsilon, \omega) \varphi(x) \mathrm{d} x \\
& =-\frac{1}{\varepsilon} \int_{\mathbb{R}^{n}} d(x / \varepsilon, \omega) \varphi(x) e^{-2 V(x / \varepsilon, \omega)} \mathrm{d} x
\end{aligned}
$$

for any smooth function $\varphi$ with compact support on $\mathbb{R}^{n}$.

It is known (see e.g., [12, Theorem 8.24, p. 202] for example) that there exist some continuous version of $x \mapsto h^{\varepsilon}(x, \omega)$ and $x \mapsto u^{\varepsilon}(x, \omega)$. So, we choose these continuous versions of $u^{\varepsilon}$ and $h^{\varepsilon}$. We assume that $u^{\varepsilon}(0, \omega)=0$ and $h^{\varepsilon}(0, \omega)=0$ for any $\varepsilon>0$. 
A. Lejay / Homogenization of divergence-form operators in random media

Proposition 2. For almost every realization $\omega$, the families of functions $\left(h^{\varepsilon}(\cdot, \omega)\right)_{\varepsilon>0}$ and $\left(v_{i}^{\varepsilon}(\cdot, \omega)\right)_{\varepsilon>0}$ converge uniformly to 0 on each compact of $\mathbb{R}^{n}$.

Proof. For almost every realization $\omega$, the functions $v$ and $h$ have a sublinear growth. More precisely, for any $R>0$, let $\overline{B(0, R)}$ be the closed ball of radius $R$ and centered on 0 . Hence $u^{\varepsilon}(\cdot, \omega)=\varepsilon v(\cdot / \varepsilon, \omega)$ and $h^{\varepsilon}(\cdot, \omega)=$ $\varepsilon h(\cdot / \varepsilon, \omega)$ converge to 0 in $\mathrm{L}^{2}(\overline{B(0, R)})$ (see e.g., [18] or [9, 8]).

In fact, standard results on elliptic PDE (see e.g., [12, Theorem 8.24, p. 202]) implies that $h^{\varepsilon}$ and $u^{\varepsilon}$ are Hölder continuous on the ball $B(0, R / 2)$ for some coefficient $\alpha$ that depends only on $\lambda, \Lambda$ and $n$. Furthermore,

$$
\left\|h^{\varepsilon}(\cdot, \omega)\right\|_{\mathcal{C}^{\alpha}(B(0, R / 2))} \leqslant C\left(\left\|h^{\varepsilon}(\cdot, \omega)\right\|_{\mathrm{L}^{2}(B(0, R))}+\|\widehat{d}\|_{\infty}\right),
$$

and $\left\|v^{\varepsilon}(\cdot, \omega)\right\|_{\mathcal{C}^{\alpha}(B(0, R / 2))} \leqslant C^{\prime}+C\left\|v^{\varepsilon}(\cdot, \omega)\right\|_{L^{2}(B(0, R))}$, where the constants $C$ and $C^{\prime}$ depend also only on $\lambda, \Lambda, R$ and $n$. With the Ascoli Theorem, $\left(v^{\varepsilon}\right)_{\varepsilon>0}$ and $\left(h^{\varepsilon}\right)_{\varepsilon>0}$ are relatively compact on the space of continuous function on $\overline{B(0, R)}$. Hence, some subsequences of $\left(v^{\varepsilon}\right)_{\varepsilon>0}$ and $\left(h^{\varepsilon}\right)_{\varepsilon>0}$ converge in this space. But, due to the sublinear growth of $h^{\varepsilon}$ and $v^{\varepsilon}$, and the choice of $v^{\varepsilon}(\omega, 0)=h^{\varepsilon}(\omega, 0)=0$, any limit of possible convergent subsequences is equal to 0 .

Corollary 1. The quantities $\sup _{t \in[0, T]}\left|v_{i}^{\varepsilon}\left(\mathbf{X}_{t}^{\varepsilon, \omega}, \omega\right)\right|$ and $\sup _{t \in[0, T]}\left|h^{\varepsilon}\left(\mathbf{X}_{t}^{\varepsilon, \omega}, \omega\right)\right|$ converge to 0 in probability.

Proof. With the Aronson estimate (5),

$$
\mathbb{P}_{0, \omega}^{\varepsilon}[\mathrm{X} \text { exits from the ball } B(0, r) \text { during }[0, T]] \leqslant \kappa \exp \left(\frac{-r^{2}}{\kappa t}\right)
$$

for some constant $\kappa$ that depends only on $\lambda, \Lambda$ and $n$ (cf. [38, Lemma II.1.2, p. 330]). Hence, for any $\kappa>0$ and any $R>0$,

$$
\begin{aligned}
\mathbb{P}_{0, \omega}^{\varepsilon}\left[\sup _{t \in[0, T]}\left|v_{i}^{\varepsilon}\left(\mathbf{X}_{t}, \omega\right)\right| \geqslant \kappa\right] & \leqslant \mathbb{P}_{0, \omega}^{\varepsilon}\left[\sup _{t \in[0, T]}\left|v_{i}^{\varepsilon}\left(\mathbf{X}_{t}, \omega\right)\right| \geqslant \kappa ; \sup _{t \in[0, T]}\left|\mathbf{X}_{t}\right| \geqslant R\right] \\
& +\mathbb{P}_{0, \omega}^{\varepsilon}\left[\sup _{t \in[0, T]}\left|v_{i}^{\varepsilon}\left(\mathbf{X}_{t}, \omega\right)\right| \geqslant \kappa ; \sup _{t \in[0, T]}\left|\mathbf{X}_{t}\right|<R\right] .
\end{aligned}
$$

Corollary 1 is then proved using Proposition 2 .

Before proving Proposition 1, we state a Lemma which allows us to pass to the limit. 
A. Lejay / Homogenization of divergence-form operators in random media

Lemma 3. If $\mathbf{k}$ is a function in $\mathrm{L}^{1}(\mu)$ and if $\mathrm{X}_{0}^{\varepsilon, \omega}=0$, then

$$
\left(\int_{\varepsilon^{2}}^{t \wedge \varepsilon^{2}} k\left(\mathbf{X}_{s}^{\varepsilon, \omega} / \varepsilon\right) \mathrm{d} s, t \geqslant 0\right) \underset{\varepsilon \rightarrow 0}{\stackrel{\text { dist. }}{\longrightarrow}}\left(t \mapsto t \int \mathbf{k} e^{-2 \mathbf{V}} \mathrm{d} \mu\right)
$$

on the space of continuous functions for almost every environment $\omega \in \Omega$.

Proof. Assuming that $\mathrm{X}_{0}^{\varepsilon, \omega}=0$, it follows from the Ergodic Theorem that

$$
\int_{\varepsilon^{2}}^{t} k\left(\mathbf{X}_{s}^{\varepsilon, \omega} / \varepsilon\right) \mathrm{d} s \stackrel{\text { dist. }}{=} \int_{1}^{t / \varepsilon^{2}} \mathbf{k}\left(\mathrm{Y}_{s}^{\omega}\right) \mathrm{d} s \underset{\varepsilon \rightarrow 0}{\longrightarrow} t \int_{\Omega} \mathbf{k} e^{-2 \mathbf{V}} \mathrm{d} \mu, \mathbb{P}_{\omega} \text {-a.s.. }
$$

for almost every $\omega$ in $\Omega$. In fact, the convergence holds uniformly in $t$ on some compact interval $[0, T]$, because $\mathbf{k}$ may be decomposed as the difference of two non-negative functions $\mathbf{k}^{+}$and $\mathbf{k}^{-}$. Thus $t \mapsto \int_{1}^{t / \varepsilon^{2}} \mathbf{k}^{+}\left(\mathrm{Y}_{s}^{\omega}\right) \mathrm{d} s$ is increasing and according to the Dini Theorem, it converges uniformly for any fixed environment.

Proof of Proposition 11. With the Itô-Fukushima decomposition [11, Theorem 5.5.1, p. 228 and Theorem 5.2.3, p. 206], $\mathbb{P}_{0, \omega}^{\varepsilon}$-almost surely,

$$
\left[\begin{array}{c}
\mathbf{X}_{t}-\mathbf{X}_{\varepsilon^{2}} \\
V_{t}^{\varepsilon, \omega}(\mathbf{X})-V_{\varepsilon^{2}}^{\varepsilon, \omega}(\mathbf{X})
\end{array}\right]+\left[\begin{array}{c}
v^{\varepsilon}\left(\mathbf{X}_{t}, \omega\right)-v^{\varepsilon}\left(\mathbf{X}_{\varepsilon^{2}}, \omega\right) \\
h^{\varepsilon}\left(\mathbf{X}_{t}, \omega\right)-h^{\varepsilon}\left(\mathbf{X}_{\varepsilon^{2}}, \omega\right)
\end{array}\right]=\mathrm{M}_{t}^{\varepsilon, \omega}+\mathrm{R}_{t}^{\varepsilon, \omega}, \forall t \geqslant \varepsilon^{2},
$$

where $\mathbf{M}^{\varepsilon, \omega}$ is a $(n+1)$-dimensional local martingale and

$$
\mathrm{R}_{t}^{i, \varepsilon, \omega}= \begin{cases}0 & \text { if } i=1, \ldots, n, \\ \int_{\varepsilon^{2}}^{t} c\left(\mathbf{X}_{s}^{\varepsilon, \omega} / \varepsilon, \omega\right) \mathrm{d} s & \text { if } i=n+1 .\end{cases}
$$

Remark 3. We start at time $\varepsilon^{2}$ to be sure that decomposition (13) holds really under $\mathbb{P}_{0, \omega}^{\varepsilon}$, which is not ensured by the results in [11] when the dimension $n$ is greater than 1 , since $\{0\}$ is a set of zero capacity.

The cross-variations of $\mathbf{M}^{\varepsilon, \omega}$ are

$$
\left\langle\mathbf{M}^{i, \varepsilon, \omega}, \mathbf{M}^{j, \varepsilon, \omega}\right\rangle_{t}=\left\{\begin{aligned}
& \int_{\varepsilon^{2}}^{t} a_{p, q}\left(\delta_{p, i}+\frac{\partial v_{i}}{\partial x_{p}}\right)\left(\delta_{q, j}+\frac{\partial v_{j}}{\partial x_{q}}\right)\left(\mathbf{X}_{s} / \varepsilon, \omega\right) \mathrm{d} s \\
& \quad \text { if } i, j=1, \ldots, n, \\
& \int_{\varepsilon^{2}}^{t} a_{p, q}\left(\delta_{p, i}+\frac{\partial v_{i}}{\partial x_{p}}\right) \frac{\partial h}{\partial x_{q}}\left(\mathbf{X}_{s} / \varepsilon, \omega\right) \mathrm{d} s \quad \text { if }(i, j) \text { and }(j, i) \text { in }\{1, \ldots, n\} \times\{n+1\}, \\
& \int_{\varepsilon^{2}}^{t} a_{p, q} \frac{\partial h}{\partial x_{p}} \frac{\partial h}{\partial x_{q}}\left(\mathbf{X}_{s} / \varepsilon, \omega\right) \mathrm{d} s \\
& \quad \text { if } i=n+1 \text { and } j=n+1 .
\end{aligned}\right.
$$


A. Lejay / Homogenization of divergence-form operators in random media

The Central Limit Theorem for the martingales (see e.g., Theorem 7.1.4 in [7, p. 339]), implies that $\mathrm{M}^{\varepsilon, \omega}$ converges to a $(n+1)$-dimensional Gaussian process $\overline{\mathrm{M}}$ whose cross-variations are

$$
\left\langle\overline{\mathbf{M}}^{i}, \overline{\mathbf{M}}^{j}\right\rangle_{t}=\left\{\begin{array}{l}
t \int_{\Omega} \mathbf{a}_{p, q}\left(\delta_{p, i}+\mathbf{f}_{p}^{i}\right)\left(\delta_{q, j}+\mathbf{f}_{q}^{j}\right) e^{-2 \mathbf{V}} \mathrm{d} \mu=t \bar{a}_{i, j} \\
\quad \text { if } i, j=1, \ldots, n, \\
t \int_{\Omega} \mathbf{a}_{p, q}\left(\delta_{p, i}+\mathbf{f}_{p}^{i}\right) \mathbf{g}_{q} e^{-2 \mathbf{V}} \mathrm{d} \mu=0 \text { from (10), } \\
\quad \text { if }(i, j) \text { and }(j, i) \text { in }\{1, \ldots, n\} \times\{n+1\}, \\
t \int_{\Omega} \mathbf{a}_{p, q} \mathbf{g}_{p} \mathbf{g}_{q} e^{-2 \mathbf{V}} \mathrm{d} \mu=t \bar{D} \\
\text { if } i=n+1 \text { and } j=n+1 .
\end{array}\right.
$$

Since $\mathrm{R}^{\varepsilon, \omega}$ converges in distribution to the function $t \mapsto(0, \ldots, 0, t \bar{c})$ with $\bar{c}=\int_{\Omega} \mathbf{c} e^{-2 \mathbf{V}} \mathrm{d} \mu$, Proposition 1 is proved, and the coefficients $\bar{a}$ and $\bar{D}$ are identified by formula (16).

\section{Addition of a first-order term}

We add now a first-order differential term to the operator $A^{\varepsilon, \omega}$.

Hypothesis 3 . The function $\mathbf{b}$ be a measurable function on $\Omega$ bounded by $\Lambda$.

The stationary random field corresponding to $\mathbf{b}$ is denoted by $b$.

Let $\left(\widetilde{L}^{\varepsilon, \omega}, \operatorname{Dom}\left(\widetilde{L}^{\varepsilon, \omega}\right)\right)$ be the operator

$$
\widetilde{L}^{\varepsilon, \omega}=L^{\varepsilon, \omega}+b_{i}(x / \varepsilon, \omega) \frac{\partial}{\partial x_{i}}, \operatorname{Dom}\left(\widetilde{L}^{\varepsilon, \omega}\right)=\operatorname{Dom}\left(L^{\varepsilon, \omega}\right) .
$$

There exists also a Feller semi-group associated to this operator, and this semi-group admit a density which also satisfies the Aronson estimate (5) and is Hölder continuous. But, in this case, these estimates (5) and (6) are only valid on any compact interval $[0, T]$ of time, for some constants that depend also on $T$ (see e.g., 38]). Thus, there exists a conservative, continuous strong Markov process $\left(\mathrm{X}, \widetilde{\mathbb{P}}_{x, \omega}^{\varepsilon}\right)_{x \in \mathbb{R}^{n}}$ whose infinitesimal generator is $\left(\widetilde{L}^{\varepsilon, \omega}, \operatorname{Dom}\left(\widetilde{L}^{\varepsilon, \omega}\right)\right)$.

For any point $x$, the Markov process $\mathrm{X}$ under $\mathbb{P}_{x, \omega}^{\varepsilon}$ may be decomposed as the sum of a martingale $\mathbf{M}^{\mathbf{X}^{\varepsilon, \omega}}$ and a continuous process $\mathrm{N}^{\varepsilon, \omega}$ locally of zero-quadratic variations [37. 
A. Lejay / Homogenization of divergence-form operators in random media

Proposition 3. For any $x$ in $\mathbb{R}^{n}$, the distribution $\widetilde{\mathbb{P}}_{x, \omega}^{\varepsilon}$ is absolutely continuous with respect to $\mathbb{P}_{x, \omega}^{\varepsilon}$, and

$$
\left.\frac{\mathrm{d} \widetilde{\mathbb{P}}_{x, \omega}^{\varepsilon}}{\mathrm{d} \mathbb{P}_{x, \omega}^{\varepsilon}}\right|_{\mathcal{F}_{t}^{\varepsilon, \omega}}=Z_{t}^{\varepsilon, \omega}
$$

where $\mathbf{Z}^{\varepsilon, \omega}$ is the exponential martingale

$$
\mathrm{Z}_{t}^{\varepsilon, \omega}=\exp \left(\widetilde{\mathrm{M}}_{t}^{\varepsilon, \omega}-\frac{1}{2}\left\langle\widetilde{\mathrm{M}}^{\varepsilon, \omega}\right\rangle_{t}\right) \text { and } \widetilde{\mathrm{M}}_{t}^{\varepsilon, \omega}=\int_{0}^{t}\left(a^{-1} b\right)^{\mathrm{t}}\left(\mathrm{X}_{s} / \varepsilon, \omega\right) \mathrm{dM}_{s}^{\mathbf{X}^{\varepsilon, \omega}}
$$

Here $\mathbf{M}^{\mathbf{X}^{\varepsilon, \omega}}$ is the martingale part of $\mathbf{X}^{\varepsilon, \omega}$ under $\mathbb{P}_{x, \omega}^{\varepsilon}$.

Sketch of the proof. As $a^{-1}$ and $b$ are bounded respectively by $\lambda$ and $\Lambda$, we deduce easily that $\mathbb{E}_{x, \omega}^{\varepsilon}\left[\exp \left(\frac{1}{2}\left\langle\widetilde{\mathrm{M}}^{\varepsilon, \omega}\right\rangle_{t}\right)\right]<e^{\lambda \Lambda^{2} t}$ for any $t \geqslant 0$, where $\Lambda$ is the upper bound of $a$ and $b$. Hence $Z^{\varepsilon, \omega}$ is an exponential martingale.

It can be proved that $\widetilde{\mathbb{E}}_{x, \omega}^{\varepsilon}\left[f\left(\mathrm{X}_{t}\right)\right]$ is equal to $\widetilde{P}^{\varepsilon, \omega} f(x)$ for almost every $x$ in $\mathrm{L}^{2}\left(\mathbb{R}^{n}\right)$ (see [26] or [5]). But, we know that $\widetilde{P}_{t}^{\varepsilon, \omega} f(x)$ is continuous, and using Proposition 3.7 in [5], $x \mapsto \widetilde{\mathbb{E}}_{x, \omega}^{\varepsilon}\left[f\left(\mathrm{X}_{t}\right)\right]$ is also continuous. Hence, for any $x \in \mathbb{R}^{n}, \widetilde{\mathbb{P}}_{x, \omega}^{\varepsilon}$ is the distribution of the process associated to the operator $\left(\widetilde{L}^{\varepsilon, \omega}, \operatorname{Dom}\left(\widetilde{L}^{\varepsilon, \omega}\right)\right)$.

Proposition 4. For any bounded function $f$ on $[0, t] \times \mathcal{C}\left([0, t] ; \mathbb{R}^{n}\right)$

$$
\mathbb{E}_{x, \omega}^{\varepsilon}\left[\mathbf{Z}_{t}^{\varepsilon, \omega} f(\cdot)\right] \leqslant e^{\Lambda^{2} \lambda t / 2} \mathbb{E}_{x, \omega}^{\varepsilon}\left[f(\cdot)^{2}\right]^{1 / 2}
$$

where $\lambda$ is the ellipticity constant of $a$, and $\Lambda$ is the bound of the coefficients a and $b$.

Proof. Using the Cauchy-Schwarz inequality, it is standard that

$$
\mathbb{E}_{x, \omega}^{\varepsilon}\left[\mathbf{Z}_{t}^{\varepsilon, \omega} f(\cdot)\right] \leqslant \mathbb{E}_{x, \omega}^{\varepsilon}\left[e^{2 \widetilde{\mathrm{M}}_{t}^{\varepsilon, \omega}-\frac{4}{2}\left\langle\widetilde{\mathrm{M}}^{\varepsilon, \omega}\right\rangle_{t}}\right]^{1 / 2} \mathbb{E}_{x, \omega}^{\varepsilon}\left[e^{\left\langle\widetilde{\mathrm{M}}^{\varepsilon, \omega}\right\rangle_{t}} f(\cdot)^{2}\right]^{1 / 2}
$$

which proves (17), since $Z^{\varepsilon, \omega}$ is an exponential martingale and $\left\langle\widetilde{M}^{\varepsilon, \omega}\right\rangle_{t}$ is bounded by $\Lambda^{2} \lambda t$.

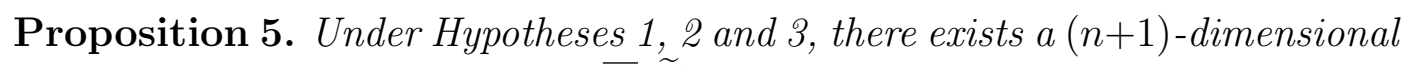
non-standard Brownian motion $(\overline{\mathrm{M}}, \widetilde{\mathbb{P}})$ with the same cross-variations as as in Proposition 1, such that for almost every environment $\omega \in \Omega$,

$$
\begin{aligned}
\mathcal{L}\left(\mathrm{X}^{1}, \ldots, \mathrm{X}^{n}, V^{\varepsilon, \omega}(\mathrm{X}) \mid \widetilde{\mathbb{P}}_{0, \omega}^{\varepsilon}\right) & \underset{\varepsilon \rightarrow 0}{\longrightarrow} \mathcal{L}\left(\overline{\mathrm{M}}_{t}^{1}+t \bar{b}_{1}, \ldots, \overline{\mathrm{M}}_{t}^{n}+t \bar{b}_{n}, \overline{\mathrm{M}}_{t}^{n+1}+t \bar{b}_{n+1}+\bar{c} t, t \geqslant 0 \mid \widetilde{\mathbb{P}}\right),
\end{aligned}
$$


where

$$
\bar{b}= \begin{cases}t \int_{\Omega} \mathbf{b}_{j}\left(\delta_{i, j}+\mathbf{f}_{j}^{i}\right) e^{-2 \mathbf{V}} \mathrm{d} \mu, & \text { if } i=1, \ldots, n, \\ t \int_{\Omega} \mathbf{b}_{j} \mathbf{g}_{j} e^{-2 \mathbf{V}} \mathrm{d} \mu, & \text { if } i=n+1 .\end{cases}
$$

Proof. We may now consider the process $\left(\mathrm{X}, V^{\varepsilon, \omega}(\mathrm{X})\right)$ under the distribution $\widetilde{\mathbb{P}}_{0, \omega}^{\varepsilon}$. It follows from the Girsanov transform that under $\widetilde{\mathbb{P}}_{0, \omega}^{\varepsilon}$, Equation (13) becomes

$$
\left[\begin{array}{c}
\mathrm{X}_{t}-\mathbf{X}_{\varepsilon^{2}} \\
V_{t}^{\varepsilon, \omega}(\mathrm{X})-V_{\varepsilon^{2}}^{\varepsilon, \omega}(\mathbf{X})
\end{array}\right]+\left[\begin{array}{c}
v^{\varepsilon}\left(\mathbf{X}_{t}, \omega\right)-v^{\varepsilon}\left(\mathbf{X}_{\varepsilon^{2}}, \omega\right) \\
h^{\varepsilon}\left(\mathrm{X}_{t}, \omega\right)-h^{\varepsilon}\left(\mathrm{X}_{\varepsilon^{2}}, \omega\right)
\end{array}\right]=\mathrm{M}_{t}^{\varepsilon, \omega}+\mathrm{R}_{t}^{\varepsilon, \omega}+\mathrm{U}_{t}^{\varepsilon, \omega}
$$

where $R^{\varepsilon, \omega}$ has been defined previously by (14), $\mathrm{M}^{\varepsilon, \omega}$ is a local martingale whose cross-variations are given by (15), and

$$
\mathrm{U}_{t}^{i, \varepsilon, \omega}= \begin{cases}\int_{\varepsilon^{2}}^{t} b_{j}\left(\delta_{i, j}+\frac{\partial v_{i}}{\partial x_{j}}\right)\left(\mathbf{X}_{s}^{\varepsilon, \omega} / \varepsilon, \omega\right) \mathrm{d} s & \text { if } i=1, \ldots, n, \\ \int_{\varepsilon^{2}}^{t} b_{j} \frac{\partial h}{\partial x_{j}}\left(\mathbf{X}_{s}^{\varepsilon, \omega} / \varepsilon, \omega\right) \mathrm{d} s & \text { if } i=n+1 .\end{cases}
$$

With (17), it is clear that the convergence in probability under $\mathbb{P}_{0, \omega}^{\varepsilon}$ to some deterministic function implies the convergence in probability to the same function under $\widetilde{\mathbb{P}}_{0, \omega}^{\varepsilon}$.

Hence, $\mathrm{M}^{\varepsilon, \omega}$ converges to some Gaussian process $\overline{\mathrm{M}}$ whose cross-variations are given by (16), since we use the almost sure convergence of its crossvariations. Furthermore, with Lemma $3, \mathrm{R}^{\varepsilon, \omega}$ converges in probability to $t \mapsto(0, \ldots, 0, t \bar{c})$, and $\mathrm{U}^{\varepsilon, \omega}$ converges to $t \mapsto t \bar{b}$.

Finally, we remark that

$$
\widetilde{\mathbb{P}}_{0, \omega}^{\varepsilon}\left[\sup _{0 \leqslant t \leqslant T}\left|v^{\varepsilon}\left(\mathbf{X}_{t}, \omega\right)\right|+\sup _{0 \leqslant t \leqslant T}\left|h^{\varepsilon}\left(\mathbf{X}_{t}, \omega\right)\right| \geqslant \eta\right] \underset{\varepsilon \rightarrow 0}{\longrightarrow} 0,
$$

for any $\eta>0$ and for almost every realization $\omega$. Proposition 5 is proved by combining all the previous convergences.

\section{Application to PDEs}

Let $\mathcal{O}$ be an open, connected subset of $\mathbb{R}^{n}$. We assume that the boundary of $\mathcal{O}$ is regular enough.

From the Theory of Dirichlet form, the process associated to the strong local, regular Dirichlet form

$$
\int_{\mathcal{O}} a_{i, j}(x / \varepsilon, \omega) \frac{\partial u(x)}{\partial x_{i}} \frac{\partial v(x)}{\partial x_{j}} e^{-2 V(x / \varepsilon, \omega)} \mathrm{d} x_{j}-\int_{\mathcal{O}} b_{i}(x / \varepsilon, \omega) \frac{\partial u(x)}{\partial x_{i}} v(x) \mathrm{d} x,
$$


defined on $\mathrm{H}_{0}^{1}(\mathcal{O}) \times \mathrm{H}_{0}^{1}(\mathcal{O})$, is the process $X^{\varepsilon, \omega}$ killed when it exits from $\mathcal{O}$ ([11, Theorem 4.2.2, p. 154] for the case where $b=0$. The Girsanov theorem also holds in this case).

Since the boundary of $\mathcal{O}$ is regular, the set of discontinuities for the function that gives the exit time of a path is of null measure with respect to the distribution $\mathbb{P}$ and $\widetilde{\mathbb{P}}$. In other words, if $O$ belongs to $\mathcal{O}$ and

$$
\mathrm{t}^{\mathcal{O}}=\inf \{t \geqslant 0 \mid \mathrm{X} \notin \mathcal{O}\} \text {, }
$$

then $\mathcal{L}\left(\mathrm{X}, V^{\varepsilon, \omega}(\mathrm{X}), \mathrm{t}^{\mathcal{O}} \mid \widetilde{\mathbb{P}}_{0, \omega}^{\varepsilon}\right)$ converges in distribution to $\mathcal{L}\left(\mathrm{X}, \overline{\mathrm{V}}, \mathrm{t}^{\mathcal{O}} \mid \widetilde{\mathbb{P}}\right)$, where

$$
\overline{\mathrm{V}}_{t}=\overline{\mathrm{M}}_{t}^{n+1}+t \bar{b}_{n+1}+\bar{c} t .
$$

\subsection{Parabolic PDE}

We are first interested in the behaviour of the solutions of the parabolic PDEs

$$
\left\{\begin{array}{l}
\frac{\partial u^{\varepsilon}(t, x, \omega)}{\partial t}=A^{\varepsilon, \omega} u^{\varepsilon}(t, x, \omega),(t, x) \in \mathbb{R}_{+} \times \mathcal{O}, \\
u^{\varepsilon}(0, x)=f(x) \in \mathrm{L}^{2}(\mathcal{O}), \\
u^{\varepsilon}(t, \cdot) \in \mathrm{H}_{0}^{1}(\mathcal{O}), \forall t>0
\end{array}\right.
$$

as $\varepsilon$ goes to 0 .

Theorem 1. We assume that Hypotheses 1, 2 and 9 are satisfied. Let $\mathcal{O}$ be a domain of $\mathbb{R}^{n}$ with regular boundary, and $f$ a function in $\mathrm{L}^{2}(\mathcal{O})$. Let $\bar{A}$ be the operator on $\mathcal{O}$ defined by

$$
\begin{gathered}
\bar{A}=\frac{1}{2} \bar{a}_{i, j} \frac{\partial^{2}}{\partial x_{i} \partial x_{j}}+\bar{b}_{i} \frac{\partial}{\partial x_{i}}+\bar{d}, \\
\operatorname{Dom}(\bar{A})=\left\{f \in \mathrm{H}_{0}^{1}(\mathcal{O}) \mid \triangle f \in \mathrm{L}^{2}(\mathcal{O})\right\},
\end{gathered}
$$

where for $i, j=1, \ldots, n+1$,

$$
\begin{gathered}
\bar{a}_{i, j}=\int_{\Omega} \mathbf{a}_{p, q}\left(\delta_{p, i}+\mathbf{f}_{p}^{i}\right)\left(\delta_{q, j}+\mathbf{f}_{q}^{j}\right) e^{-2 \mathbf{V}} \mathrm{d} \mu, \bar{b}_{i}=\int_{\Omega} \mathbf{b}_{j}\left(\delta_{i, j}+\mathbf{f}_{j}^{i}\right) e^{-2 \mathbf{V}} \mathrm{d} \mu, \\
\text { and } \bar{d}=\int_{\Omega}\left(\frac{1}{2} \mathbf{a}_{i, j} \mathbf{g}_{i} \mathbf{g}_{j}+\mathbf{c}+\mathbf{b}_{i} \mathbf{g}_{i}\right) e^{-2 \mathbf{V}} \mathrm{d} \mu
\end{gathered}
$$

for $\mathbf{f}_{j}^{i}$ and $\mathbf{g}_{j}$ defined in (10) and (11). Then a version of the generalized solution to the parabolic equation (19) converges pointwise for any $(t, x) \in$ $\mathbb{R}_{+} \times \mathcal{O}$ to the solution $\bar{u}$ of the parabolic PDE

$$
\left\{\begin{array}{l}
\frac{\partial \bar{u}(t, x)}{\partial t}=\bar{A} \bar{u}(t, x), \quad(t, x) \in \mathbb{R}_{+} \times \mathcal{O} \\
\bar{u}(t, x) \in \mathrm{H}_{0}^{1}(\mathcal{O}), \forall t>0 \\
\bar{u}(0, x)=f(x)
\end{array}\right.
$$


A. Lejay / Homogenization of divergence-form operators in random media

A version of the generalized solution $u^{\varepsilon}$ of this equation is given by the Feynman-Kac formula:

$$
u^{\varepsilon}(t, x, \omega)=\widetilde{\mathbb{E}}_{x, \omega}^{\varepsilon}\left[\exp \left(V_{t}^{\varepsilon, \omega}(\mathrm{X})\right) f\left(\mathrm{X}_{t}\right) ; t<\mathrm{t}^{\mathcal{O}}\right]
$$

where $\mathrm{V}^{\varepsilon, \omega}$ has been defined in (9). From the stationarity of the coefficients of the operator $A^{1, \omega}$, the density transition function $p^{\varepsilon}(\omega, t, x, y)$ satisfies

$$
p^{\varepsilon}(\omega, t, x, y)=p^{\varepsilon}\left(\tau_{x / \varepsilon} \omega, t, 0, y-x\right), \forall \varepsilon>0, \forall x, y \in \mathbb{R}^{n} .
$$

As $\left(\tau_{x}\right)_{x \in \mathbb{R}^{n}}$ is stationary,

$$
\begin{aligned}
u^{\varepsilon}(t, x, \omega) & =\widetilde{\mathbb{E}}_{0, \tau_{x / \varepsilon} \omega}^{\varepsilon}\left[\exp \left(V_{t}^{\varepsilon, \tau_{x / \varepsilon} \omega}(\mathrm{X})\right) f\left(\mathrm{X}_{t}+x\right) ; t<\mathrm{t}^{\mathcal{O}-x}\right] \\
& \stackrel{\text { dist. }}{=} \widetilde{\mathbb{E}}_{0, \omega}^{\varepsilon}\left[\exp \left(V_{t}^{\varepsilon, \omega}(\mathrm{X})\right) f\left(\mathrm{X}_{t}+x\right) ; t<\mathrm{t}^{\mathcal{O}-x}\right] .
\end{aligned}
$$

We first remark that the family $\left(\exp \left(V_{t}^{\varepsilon, \omega}\left(\mathbf{X}^{\varepsilon, \omega}\right)\right)\right)_{\varepsilon>0}$ is uniformly integrable.

Lemma 4. Under the probability $\int_{\Omega} \widetilde{\mathbb{P}}_{0, \omega}^{\varepsilon}[\cdot] \mathrm{d} \mu(\omega)$, the family $\left(\exp \left(V_{t}^{\varepsilon, \omega}\left(\mathbf{X}^{\varepsilon, \omega}\right)\right)\right)_{\varepsilon>0}$ is uniformly integrable. In fact,

$$
\sup _{\varepsilon>0} \int_{\Omega} \widetilde{\mathbb{E}}_{0, \omega}^{\varepsilon}\left[\exp \left(2 \int_{0}^{t} \frac{1}{\varepsilon} d\left(\mathbf{X}_{s} / \varepsilon, \omega\right) \mathrm{d} s\right)\right] \mathrm{d} \mu(\omega)<+\infty .
$$

The following Lemma will be used.

Lemma 5. There exists some constant $C$ depending only on the bounds of $\widehat{\mathbf{d}}_{1}, \ldots, \widehat{\mathbf{d}}_{n}$ such that

$$
\left|\int_{\Omega} e^{-2 \mathbf{V}} \mathbf{d u}^{2} \mathrm{~d} \mu\right| \leqslant C\|\mathbf{u}\|_{\mathrm{L}^{2}(\mu)}\|\nabla \mathbf{u}\|_{\mathrm{L}^{2}(\mu)^{n}}
$$

for any bounded function $\mathbf{u}$ in $\mathcal{H}^{1}(\mu)$.

Proof. Let $\mathbf{u}$ be a bounded function in $\mathcal{H}^{1}(\mu)$. A straightforward calculation implies that

$$
D_{i}\left(\mathbf{u}^{2}\right)=2 \mathbf{u} D_{i} \mathbf{u}, \text { for } i=1, \ldots, n \text {. }
$$

So,

$$
\left|\int_{\Omega} \mathbf{d} e^{-2 \mathbf{V}} \mathbf{u}^{2} \mathrm{~d} \mu\right|=\left|\int_{\Omega} \widehat{\mathbf{d}}_{i} D_{i}\left(\mathbf{u}^{2}\right) \mathrm{d} \mu\right| \leqslant 2 \sup _{i=1 \ldots, n}\left\|\widehat{\mathbf{d}}_{i}\right\|_{\infty} \sum_{i=1}^{n}\left|\int_{\Omega} \mathbf{u} D_{i} \mathbf{u} \mathrm{d} \mu\right|
$$

and the Lemma is proved with the Cauchy-Schwarz Inequality. 
A. Lejay / Homogenization of divergence-form operators in random media

Proof of Lemma 4. To prove (21), we use an argument borrowed from Chapter 3 in [29].

Let $\mathbf{u}^{\varepsilon}(t, \omega)$ be the solution to the parabolic equation

$$
\frac{\partial \mathbf{u}^{\varepsilon}(t, \omega)}{\partial t}=\frac{e^{2 \mathbf{V}}}{2} D_{i}\left(e^{-2 \mathbf{V}} \mathbf{a}_{i, j} D_{j} \mathbf{u}^{\varepsilon}\right)(t, \omega)+4 \varepsilon \mathbf{d} \mathbf{u}^{\varepsilon}(t, \omega)
$$

with the initial condition $\mathbf{u}^{\varepsilon}(0, \omega)=1$. This solution $\mathbf{u}^{\varepsilon}(t, \cdot)$ belongs to $\mathcal{H}^{1}(\mu)$ for any $t \geqslant 0$, and is given by the Feynman-Kac formula:

$$
\mathbf{u}^{\varepsilon}(t, \omega)=\mathbb{E}_{\omega}\left[\exp \left(4 \varepsilon \int_{0}^{t} \mathbf{d}\left(\mathrm{Y}_{s}^{\omega}\right) \mathrm{d} s\right)\right]
$$

so that $\omega \mapsto \mathbf{u}^{\varepsilon}(t, \omega)$ is bounded for any $t \geqslant 0$ and any $\varepsilon>0$.

We know that $\mathcal{E}^{\pi}(\mathbf{u}, \mathbf{u}) \geqslant \kappa\|\nabla \mathbf{u}\|_{\mathrm{L}^{2}(\mu)^{n}}^{2}$ for $\kappa=\lambda e^{-2 \Lambda}$. It follows from (22) that

$$
\begin{aligned}
\frac{\partial}{\partial t}\left\|\mathbf{u}^{\varepsilon}(t, \cdot)\right\|_{\mathrm{L}^{2}(\pi)}^{2} & \leqslant \sup _{\substack{\mathbf{v} \in \mathrm{L}^{2}(\mu),\|\mathbf{v}\|_{\mathrm{L}^{2}(\mu)}=1}}\left\{-\kappa\|\nabla \mathbf{v}\|_{\mathrm{L}^{2}(\mu)^{n}}^{2}+\varepsilon C\|\nabla \mathbf{v}\|_{\mathrm{L}^{2}(\mu)^{n}}\right\}\left\|\mathbf{u}^{\varepsilon}(t, \cdot)\right\|_{\mathrm{L}^{2}(\mu)}^{2} \\
& \leqslant \frac{C^{2} \varepsilon^{2}}{4 \kappa} e^{4 \Lambda}\left\|\mathbf{u}^{\varepsilon}(t, \cdot)\right\|_{\mathrm{L}^{2}(\pi)}^{2}
\end{aligned}
$$

The Gronwall inequality [7, Theorem A.5.1, p. 598] yields

$$
\left\|\mathbf{u}^{\varepsilon}(t, \omega)\right\|_{L^{2}(\pi)}^{2} \leqslant \exp \left(\frac{\varepsilon^{2} t C^{2}}{2 \kappa}\right)
$$

so that, from the Feynman-Kac formula applied to the solution of (22) and the Cauchy-Schwarz Inequality,

$$
\forall \varepsilon>0, \int_{\Omega} \mathbb{E}_{\omega}\left[\exp \left(\varepsilon \int_{0}^{t / \varepsilon^{2}} 4 \mathbf{d}\left(\mathrm{Y}_{s}^{\omega}\right) \mathrm{d} s\right)\right] \mathrm{d} \mu \leqslant \exp \left(\frac{t C^{2}}{2 \kappa}\right) .
$$

With (17), there exists some constant $C$ depending only on $\lambda, \Lambda$ and $t$ such that

$$
\begin{aligned}
\widetilde{\mathbb{E}}_{x, \omega}^{\varepsilon}\left[\exp \left(\frac{2}{\varepsilon} \int_{0}^{t} d\left(\mathbf{X}_{s} / \varepsilon, \omega\right) \mathrm{d} s\right)\right] & \\
& \leqslant C \mathbb{E}_{x, \omega}^{\varepsilon}\left[\exp \left(\frac{4}{\varepsilon} \int_{0}^{t} d\left(\mathbf{X}_{s} / \varepsilon, \omega\right) \mathrm{d} s\right)\right]^{1 / 2} .
\end{aligned}
$$

Hence (21) is satisfied. 
A. Lejay / Homogenization of divergence-form operators in random media

With Hypothesis 2, there exists some bounded stationary random fields $\widetilde{d}_{1}, \ldots, \widetilde{d}_{n}$ such that

$$
\begin{aligned}
A^{\varepsilon, \omega}=L^{\varepsilon, \omega}+b_{i}(x / \varepsilon, \omega) & \frac{\partial}{\partial x_{i}}-\widetilde{d}_{i}(x / \varepsilon, \omega) \frac{\partial}{\partial x_{i}}+c(x / \varepsilon, \omega) \\
& +e^{2 V(x / \varepsilon, \omega)} \frac{\partial}{\partial x_{i}}\left(e^{-2 V(x / \varepsilon, \omega)} \widetilde{d}_{i}(x / \varepsilon, \omega) \cdot\right) .
\end{aligned}
$$

We may assume that $e^{2 V}$ and $\widetilde{d}_{1}, \ldots, \widetilde{d}_{n}$ are bounded by $\Lambda$.

The expression (25) is in fact particularly suitable, since in this case, the semi-group of infinitesimal generator $A^{\varepsilon, \omega}$ (which is in general not Markovian) also has a density transition function satisfying the Aronson estimate similar to (5) with some constants depending only on $\lambda$ and the upper bound of the coefficients (see [2]).

Proof of Theorem 1. If $f$ is continuous, bounded with compact support on $\mathcal{O}$, then $f\left(\mathbf{X}_{t}+x\right) \mathbb{1}_{\left\{t<t^{\mathcal{O}-x}\right\}}=f\left(\mathbf{X}_{t \wedge \mathfrak{t} \mathcal{O}-x}+x\right) \widetilde{\mathbb{P}}_{0, \omega}^{\varepsilon}$-a.s. because $f$ is equal to 0 on the boundary of $\mathcal{O}$. It is now clear that

$$
\begin{aligned}
\int_{\Omega} \mid & \widetilde{\mathbb{E}}_{0, \omega}^{\varepsilon}\left[\exp \left(V_{t}^{\varepsilon, \omega}\left(\mathbf{X}^{\varepsilon, \omega}\right)\right) f\left(\mathrm{X}_{t}+x\right) ; t<\mathrm{t}^{\mathcal{O}-x}\right] \\
& -\widetilde{\mathbb{E}}\left[\exp \left(\overline{\mathrm{M}}_{t}^{n+1}+\bar{b}_{n+1} t+\bar{c} t f\left(\mathbf{X}_{t}+x\right) ; t<\mathrm{t}^{\mathcal{O}-x}\right)\right] \mid \mathrm{d} \mu(\omega) \underset{\varepsilon \rightarrow 0}{\longrightarrow} 0 .
\end{aligned}
$$

It remains to remark that, using the Girsanov Theorem with the density $\mathrm{d} \widehat{\widetilde{\mathbb{P}}} /\left.\mathrm{d} \widetilde{\mathbb{P}}\right|_{\mathcal{F}_{t}}=\exp \left(\overline{\mathrm{M}}_{t}^{n+1}-\frac{1}{2}\left\langle\overline{\mathrm{M}}^{n+1}\right\rangle_{t}\right)$,

$$
\begin{aligned}
\widetilde{\mathbb{E}}\left[\operatorname { e x p } \left(\overline{\mathrm{M}}_{t}^{n+1}\right.\right. & \left.\left.+\bar{b}_{n+1} t+\bar{c} t\right) f\left(\mathrm{X}_{t}+x\right) ; t<\mathrm{t}^{\mathcal{O}-x}\right] \\
= & \widehat{\mathbb{E}}\left[\exp \left(\frac{1}{2}\left\langle\overline{\mathrm{M}}^{n+1}\right\rangle_{t}+\bar{b}_{n+1} t+\bar{c} t\right) f\left(\mathrm{X}_{t}+x\right) ; t<\mathrm{t}^{\mathcal{O}-x}\right],
\end{aligned}
$$

where, for any $t \geqslant 0$ and for $i=1, \ldots, n$,

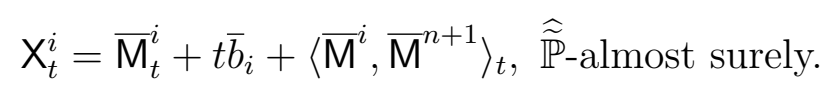

But we have seen in (16) that $\left\langle\overline{\mathrm{M}}^{i}, \overline{\mathrm{M}}^{n+1}\right\rangle_{t}=0$ for any $t \geqslant 0$. So it has been proved that

$$
\forall(t, x) \in \mathbb{R}_{+} \times \mathcal{O}, \int_{\Omega}\left|u^{\varepsilon}(t, x, \omega)-\bar{u}(t, x)\right| \mathrm{d} \mu(\omega) \underset{\varepsilon \rightarrow 0}{\longrightarrow} 0 .
$$

Let $\left(\widetilde{P}_{t}^{\varepsilon, \omega}\right)_{t>0}$ be the semi-group whose infinitesimal generator is $A^{\varepsilon, \omega}$. According to Lemma 4.1 in [36, p. 147], For any function $f$ in $\mathrm{L}^{2}(\mathcal{O})$, a subsequence of $\left(\widetilde{P}_{t}^{\varepsilon, \omega} f\right)_{\varepsilon>0}$ is convergent in $\mathrm{L}^{2}(] 0, T[\times \mathcal{O})$ for any $t>0$. If moreover $f$ is a smooth function with compact support, then $(t, x) \mapsto \widetilde{P}_{t}^{\varepsilon, \omega} f(x)$ 
converges uniformly on each compact of $] 0, T[\times \mathcal{O}$. Using the Aronson estimate for the density transition function of $\widetilde{P}^{\varepsilon, \omega}$, it is clear that this sequence is bounded on $\mathcal{O}$ for any fixed $t$ by some constant that does not depends on $\omega$. Let $u(t, x, \omega)$ be the limit of a converging subsequence of $\left(\widetilde{P}_{t}^{\varepsilon, \omega} f(x)\right)_{\varepsilon>0}$. Then the convergence also holds in $\mathrm{L}^{1}(\Omega, \mu)$, and $u(t, x, \omega)$ is almost surely equal to the limit $\bar{u}(t, x)$ when the initial condition of the parabolic PDE (20) is $f$.

Again with the Aronson estimate (5) and with the Cauchy-Schwartz inequality, $\left|\widetilde{P}_{t}^{\varepsilon, \omega} f(x)\right|$ is bounded by $e^{C t}\|f\|_{\mathrm{L}^{2}(\mathcal{O})} / t^{n / 2}$. So, by density, $\widetilde{P}_{t}^{\varepsilon, \omega} f(x)$ converges pointwise to $\bar{u}(t, x)$ for any $f$ in $\mathrm{L}^{2}(\mathcal{O})$, assuming we have chosen the version of $\widetilde{P}_{t}^{\varepsilon, \omega} f$ given using the density transition function.

Corollary 2. For any starting point $x$ and for almost every realization $\omega$, $\widetilde{\mathbb{P}}_{x, \omega}^{\varepsilon}$ converges weakly to $\widetilde{\mathbb{P}}[x+\cdot]$.

Proof. See Theorem 4.2 in [36, p. 148].

\subsection{Elliptic PDE}

Let us start with the definition of some convergence on a family of operators.

Definition 2 (G-convergence). A family $\left(B^{\varepsilon}\right)_{\varepsilon>0}$ of operators from $\mathrm{V}$ to $\mathrm{V}^{\prime}$ satisfying uniformly in $\varepsilon$

$$
\begin{gathered}
\left\langle B^{\varepsilon} u, u\right\rangle_{\mathrm{L}^{2}(\mathcal{O})} \geqslant C\|u\|_{\mathrm{H}_{0}^{1}(\mathcal{O})} \\
\text { and }\left\langle B^{\varepsilon} u, v\right\rangle_{\mathrm{L}^{2}(\mathcal{O})} \geqslant C^{\prime}\|u\|_{\mathrm{H}_{0}^{1}(\mathcal{O})}\|v\|_{\mathrm{H}_{0}^{1}(\mathcal{O})}
\end{gathered}
$$

for some constants $C$ and $C^{\prime}$ is said to be $G$-convergent to some operator $B$ if the family of solutions $\left(u^{\varepsilon}\right)_{\varepsilon>0}$ of

$$
u^{\varepsilon} \in \mathrm{V} \text { and } B^{\varepsilon} u^{\varepsilon}(x)=f(x), f \in \mathrm{V}^{\prime}
$$

converges weakly to the solution $u \in \mathrm{V}$ of the problem $B u=f$.

In fact, a family $\left(B^{\varepsilon}\right)_{\varepsilon>0}$ of operators satisfying (26) and (27) has a convergent subsequence in the $G$-topology. (see e.g., [39, 40] or [15, Chapter 5.3, p. 160]).

Remark 4. In the cited articles or books, the $G$-convergence is defined when the domain $\mathcal{O}$ is bounded. But the results may be generalized to unbounded domains (see Theorem 20 in [39, p. 108]). 
In view of (1), it is not clear that the operator $\alpha-A^{\varepsilon, \omega}$ is invertible for some $\alpha$ larger than some $\alpha_{0}$ independent from $\varepsilon$ and $\omega$. Again under Hypothesis 2, the form (25) is suitable for elliptic PDEs, since it allows to assert that there exist some constants $C$ and $C^{\prime}$ depending only on $\lambda, \Lambda$ and $\alpha$ such that (26) and (27) are true for $\alpha-A^{\varepsilon, \omega}$ [36]. Hence $\alpha-A^{\varepsilon, \omega}$ is invertible when $\alpha$ is greater than some $\alpha_{0}$ depending only on the ellipticity constant $\lambda$ and the bound $\Lambda$ of the coefficients.

In particular, the family $\left(\alpha-A^{\varepsilon, \omega}\right)_{\varepsilon>0}$ is relatively compact of $G$-convergence, when $\alpha \geqslant \alpha_{0}$.

Theorem 2. Under Hypotheses 1, 2 and 3, for almost every random environment $\omega,\left(\alpha-A^{\varepsilon, \omega}\right)_{\varepsilon>0} G$-converges as $\varepsilon$ goes to 0 .

Proof. See Theorem 5.4 in [36].

Using the results in [5], it is also possible to prove the convergence of the solutions of the non-homogeneous Dirichlet problem. In fact, when $b=c=$ $d=0$, the Convergence of Arbitrary Solutions Theorem [15, Theorem 5.2, p. 151] yields that the solution of $\left(\alpha-A^{\varepsilon, \omega}\right) u^{\varepsilon}=f$ will converges to the solution of $(\alpha-\bar{A}) u=f$, whatever the boundary condition is, assuming that $\mathcal{O}$ is bounded.

It is also possible to define similarly as the previous one a notion of $G$ convergence for the parabolic operators $\frac{\partial}{\partial t}+\left(\alpha-A^{\varepsilon, \omega}\right)$. The $G$-convergence of the elliptic operators $\left(\alpha-A^{\varepsilon, \omega}\right)_{\varepsilon, \omega}$ implies the convergence of the parabolic operators [40].

Remark 5. In the cited article [2, 39, 40, 38, 36], it is assume that $V=0$, but all the results may be easily adapted to the case of $V \neq 0$.

\section{A few variations of the above results}

Slow variation in the coefficients. It is clear that our results may be easily extended to deal with some operators of the form

$$
L^{\varepsilon, \omega}+b(x / \varepsilon, x) \frac{\partial}{\partial x_{i}}+c(x, x / \varepsilon)+\frac{1}{\varepsilon} d(x / \varepsilon),
$$

where the functions $b(\omega, x, y)=\mathbf{b}\left(\tau_{x} \omega, y\right)$ and $c(\omega, x, y)=\mathbf{c}\left(\tau_{x} \omega, y\right)$, and $y \mapsto \mathbf{b}(\cdot, y)$ and $y \mapsto \mathbf{c}(\cdot, y)$ are equi-continuous. 
A. Lejay / Homogenization of divergence-form operators in random media

Influence of the first-order term. Let $f(\varepsilon)$ be a function such that $f(\varepsilon)$ converges to 0 with $\varepsilon$. The homogenization property for the family of operators

$$
L^{\varepsilon, \omega}+f(\varepsilon) b(x / \varepsilon, \omega)
$$

is reduced to the homogenization property of the family of operators $\left(L^{\varepsilon, \omega}\right)_{\varepsilon>0}$.

Influence of the highly oscillatory zero-order term. Let $\mathbf{d}$ be a bounded measurable function, and $\langle\mathbf{d}\rangle=\int_{\Omega} \mathbf{d} e^{-2 \mathbf{V}} \mathrm{d} \mu$. We assume that $\mathbf{d}-\langle\mathbf{d}\rangle$ satisfies the Hypothesis 2. Let $u^{\varepsilon}(t, \omega, x)$ be the solution to the parabolic equation

$$
\frac{\partial u^{\varepsilon}(t, x, \omega)}{\partial t}=\left(L^{\varepsilon, \omega}+\frac{f(\varepsilon)}{\varepsilon} d(x / \varepsilon, \omega)+c(x / \varepsilon)\right) u^{\varepsilon}(t, x, \omega)
$$

where $f(\varepsilon)$ is a function that converges to 0 with $\varepsilon$. Hence, it is clear that

$$
\exp \left(\frac{-t f(\varepsilon)}{\varepsilon}\langle\mathbf{d}\rangle\right) u^{\varepsilon}(t, x, \omega)
$$

converges in $\mu$-probability to the solution $\bar{u}$ of the parabolic PDE

$$
\frac{\partial \bar{u}(t, x)}{\partial t}=\left(\frac{\bar{a}_{i, j}}{2} \frac{\partial^{2}}{\partial x_{i} \partial x_{j}}+\bar{b}_{i} \frac{\partial}{\partial x_{i}}+\int_{\Omega} \mathbf{c} e^{-2 \mathbf{V}} \mathrm{d} \mu\right) \bar{u}(t, x) .
$$

Hence, the speed $f(\varepsilon)=1$ is the first at which the highly-oscillatory zeroorder term $\frac{f(\varepsilon)}{\varepsilon} d(x / \varepsilon, \omega)$ change the operator in the limit.

Acknowledgment. The author wishes to thank the referee and Professor Étienne Pardoux for the attention they paid to this article, and Fabienne Castell for her fruitful remark on the highly oscillating zero-order term.

\section{References}

[1] C. Apelian, R.L. Holmes and M. Avellaneda. A Turbulent Transport Model: Streamline Results for a Class of Random Velocity Fields in the Plan. Communications on Pure and Applied Mathematics, 50:11, 1053-1088 (1997). 4

[2] D.G. Aronson. Non-negative solutions of linear parabolic equation. Annali della Scuola Normale Superiore di Pisa, 22, 607-693 (1968). 7, 20, 22

[3] A. Bensoussan, J.L. Lions and G. Papanicolaou. Asymptotic Analysis for Periodic Structures. North-Holland, (1978). 3 
A. Lejay / Homogenization of divergence-form operators in random media

[4] R.M. Blumenthal and R.K. Getoor. Markov Process and Potential Theory. Academic Press, (1968). 8

[5] Z.Q. Chen and Z. Zhao. Diffusion Processes and Second Order Elliptic Operators with Singular Coefficients for Lower Order Terms. Mathematische Annalen, 302, 323-357 (1995). 15, 22

[6] A. De Masi, P.A. Ferrari, S. Goldstein and W.D. Wick. An Invariance Principle for Reversible Markov Processes. Applications to Random Motions in Random Environments. Journal of Statistical Physics, 55:3/4, (1989). 3, 4

[7] S.N. Ethier and T.G. Kurtz. Markov Processes, Characterization and Convergence. Wiley (1986). 8, 14, 19,

[8] A. Fannjiang and T. Komorowski. A Martingale Approach to Homogenization of Unbounded Random Flows. The Annals of Probability, 25:4, 1872-1894 (1997). 12

[9] C.A. Fannjiang and G.C. Papanicolaou. Diffusion in Turbulence. Probability Theory and Related Fields, 105, 279-334 (1996). 3, 4, 12

[10] M. Freidlin. The Dirichlet problem for an equation with periodic coefficients depending on a small parameter. Teor. Veoj. i Primenen, 9, 133-139 (1964). 3

[11] M. Fukushima, Y. Oshima and M. Takeda. Dirichlet Forms and Symmetric Markov Process. De Gruyter (1994). 4, 8, 13, 17

[12] D. Gilbarg and N.S. Trudinger. Elliptic Partial Differential Equation of Second Order. Springer-Verlag (1977). [11, 12

[13] S. Goldstein. Antisymmetric functionals of reversible Markov processes. Annales de l'Institut Henri Poincaré, 31:1, 177-190 (1995). 3

[14] M.B. Isichenko. Percolation, Statistical topography, and transport in random media. Reviews of Modern Physics, 64:4, 961-1043 (1992). 4

[15] V.V. Jikov, S.M. Kozlov and O.A. Oleinik. Homogenization of Differential Operators and Integral Functionals. Springer-Verlag (1994). 3, 11, 21, 22

[16] C. Kipnis and S.R.S. Varadhan. Central Limit Theorem for Additive Functionnals of Reversible Markov Processes and Applications to Simple Exlusion. Communications in Mathematical Physics, 104, 1-19 (1986). 3 
A. Lejay / Homogenization of divergence-form operators in random media

[17] S.M. Kozlov. Averaging random structures. Soviet Math. Dokl., 19:4, 950-954 (1978). 3

[18] S.M. KozLov. Averaging of random operators. Math. USSR Sbornik, 37:2, 167-180 (1980). 3, 12

[19] S.M. KozLov. The Method of Averaging and Walk in Inhomogeneous Environment. Russian Math. Survey, 40:2, 73-145 (1985). 3

[20] H. Kunita. Stochastic flows and stochastic differential equations. Cambridge University Press (1990). 9

[21] O.A. Ladyženskaja, V.A. Solonnikov and N.N. URal'Ceva. Linear and Quasilinear Equations of Parabolic Type. American Mathematical Society (1968). 7

[22] C. Landim, S. Olla and H.T. Yau. Convection-diffusion Equation with Space-Time Ergodic Random Flow. Probability Theory and Related Fields, 112:2, (1998). 4, 6

[23] J. L. Lebowitz and H. Rost. The Einstein relation for the displacement of a test particle in a random environment. Stochastic Processes and their Applications, 54, 183-196 (1994). 3

[24] A. LeJAy. Méthodes probabilistes pour l'homogénéisation des opérateurs sous forme divergence: cas linéaires et semi-linéaires. Ph.D. thesis, Université de Provence (Marseille, France), 2000. 3

[25] J. Lunt, T.J. Lyons and T. Zhang. Integrability of Functionals of Dirichlet Processes, Probabilistic Representations of Semigroups and Estimates of Heat Kernel. Journal of Functional Analysis, 153, 320-342 (1998).

[26] T.J. Lyons and T. Zhang. Convergence of Non-symmetric Dirichlet Processes. Stochastics and stochastics reports, 57, 159-167 (1996). 15

[27] Z. MA and M. RöCKNER. Introduction to the Theory of (NonSymmetric) Dirichlet Forms. Universitext, Springer-Verlag (1991). 8

[28] K. OelschläGer. Homogenization of diffusion processes in a divergence free random field. The Annals of Probability, 16, 1084-1126 (1988). 4 
A. Lejay / Homogenization of divergence-form operators in random media

[29] S. Olla. Homogenization of Diffusion Processes in Random Fields. Cours de l'École doctorale de l'École Polytechnique, École Polytechnique (Palaiseau, France) (1994). 3, 4, 9, 19]

[30] H. Osada Homogenization of diffusion with random stationary coefficients. Probability Theory and Mathematical statistics, Proc. $4^{\text {th }}$ URSSJapan Symposium, Tbilisi/USSR 1982, Lecture Notes in Mathematics (Springer-Verlag), 1021, 507-517 (1983). 3

[31] H. Osada. An invariance principle for Markov processes and Brownian particles with singular interaction. Annales de l'Institut Henri Poincaré, 34:2, 217-248 (1998). 3

[32] H. Osada and T. SAitoh. An invariance principle for non-symmetric Markov process and reflecting diffusions in random domains. Probability Theory and Related Fields, 101, 45-63 (1995).

[33] É. Pardoux. Homogenization of Linear and Semilinear Second Order PDEs with Periodic Coefficients: a Probabilistic Approach. Journal of Functional Analysis, 167:2, 498-520 (1999).

[34] G.C. Papanicolau and S.R.S. Varadhan. Boundary Value Problems with Rapidly Oscillating Random Coefficients. In Random Fields, edited by J. Fritz, J.L. Lebowitz and D. SzÁsz, Colloque Math. Soc. János Bolyai 27 (North-Holland), 2, 835-873 (1979). 3, 4

[35] G.C. Papanicolaou. Diffusion in random media. In Surveys in Applied Mathematics, edited by J.B. Keller, D.W. Mclaughlin and G.C. Papanicolaou (Plenum Press), 1, 205-253 (1995). 3

[36] A. Rozkosz. Weak Convergence of Diffusions Corresponding to Divergence Form Operator. Stochastics and stochastics reports, 57, 129-157 (1996). 20, 21, 22

[37] A. Rozkosz. Stochastic Representation of Diffusions Corresponding to Divergence Form Operators, Stochastic Processes and their Applications, 63, pp. 11-33, (1996). 14

[38] D.W. Stroock. Diffusion Semigroups Corresponding to Uniformly Elliptic Divergence Form Operator. In Séminaire de Probabilités XXII, Lecture Notes in Mathematics (Springer-Verlag), 1321, 316-347 (1988). 17, 12, 14, 22 
A. Lejay / Homogenization of divergence-form operators in random media

[39] V.V. Zhikov, S.M. Kozlov, O.A. Oleinik and K. T'en NgoAn. Averaging and $G$-Convergence of Differential Operators Russian Math. Survey, 34:5, 69-147 (1979). 21, 22

[40] V.V. Zhikov, S.M. Kozlov and O.A. Oleinik. G-Convergence of Parabolic Operators. Russian Math. Survey, 36:1, 9-60 (1981). 21, 22 